\title{
A staggered-grid lowrank finite-difference method for elastic wave extrapolation
}

\author{
Qizhen $\mathrm{Du}^{1,2,3}$, Jing Ba ${ }^{*}, 4$, Dong Han ${ }^{5}$, Pengyuan Sun ${ }^{6}$, Jianlei Zhang ${ }^{6}$ \\ (1) Key laboratory of deep oil and gas, China University of Petroleum (East China), Changjiang West Road $66^{\text {th }}$, Qingdao, \\ China, 266580 \\ (2) Laboratory for Marine Mineral Resources, Qingdao National Laboratory for Marine Science and Technology, Qingdao, \\ China, 266071 \\ (3) Key Laboratory of Geophysical Prospecting, CNPC, China University of Petroleum (East China) Changjiang West Road \\ $66^{\text {th }}$, Qingdao, China, 266580 \\ (4) School of Earth Sciences and Engineering, Hohai University, Nanjing, Jiangsu, China, 211100 \\ (5) Sinopec Geophysical Research Institute, Nanjing, Jiangsu, China, 211103 \\ (6) China National Petroleum Corp Bureau of Geophysical Prospecting Inc. Zhuozhou, Hebei, China, 072750
}

Article history: received May 8, 2019; accepted December 18, 2019

\begin{abstract}
Elastic wave extrapolation in the time domain is significant for an elastic wave equation-based processing. To improve the simulation reliability and accuracy of decoupled elastic P- and Swaves, we propose the staggered-grid lowrank finite-difference method based on the elastic wave decomposition. For elastic wave propagation, a lowrank finite-difference method based on the staggered grid is derived to improve the accuracy. Regarding the application of the decoupled elastic wave equation, we derive the finite-difference scheme coefficients which are dependent on velocity. Based on the elastic wave decomposition and plane wave theories, we formulate the elastic wave-extrapolation operators, which contain trigonometric adjustment factors. Accordingly, by applying the lowrank method to approximating the operators, the finitedifference scheme is designed to discretize the decoupled wave equation. The derivation processing implies the combination of elastic wave-mode decomposition and extrapolation. The proposed method enables elastic P- and S-waves to extrapolate in the time-space domain separately and produces accurate $\mathrm{P}$-and S-wave components simultaneously. Dispersion analysis suggests that our proposed method is reliable and accurate in a wide range of wavenumber. Numerical simulation tests on a simple model and the Marmousi2 model validate the accuracy and effectiveness of the method, showing its ability in handling complex structures. Although the operators are accurate only when the medium is homogeneous, they are of high accuracy when the velocity gradient is quite small and are applicable when the velocity gradient is large. The subsequent results of reverse time migration for the Marmousi2 model also suggest that the proposed method is enough to serve as an extrapolator in-elastic reverse time migration.
\end{abstract}

Keywords: Lowrank finite-difference, Lowrank approximation, Elastic wavefield extrapolation. 


\section{Qizhen Du et al.}

\section{Introduction}

Over the past few decades, seismic exploration technique has been extended to the elastic wavefield. This remarkable stride benefits from the development of multi-component seismic acquisition technique [Granger et al., 2005; Robertsson et al., 2008], which provides the accessibility to not only the primary P-wave but also the S-wave. A great deal of information from diverse wave modes exist in multicomponent data, which portray the prominent subsurface structure and discriminate the lithological variation as compared to conventional single-component Pwave data [Stewart et al., 2003; Yan and Xie, 2012]. In such case, S-wave becomes an important factor to calibrate the bright-spot and to estimate the fractures [Du et al., 2012].

The processing based on the elastic wave equations act as a bridge between multicomponent data and elegant profiles. The subsurface wavefield consists of the P- and S-waves. For a physical meaning of P- and S-waves, the processing is commonly observed to contain either the wavefield separation [Yan and Sava, 2008; Du et al., 2012, 2014] or the wavefield decomposition [Zhang and McMechan, 2010; Wang and McMechan, 2015]. As for the separation in isotropic media, classic curl and divergence operators are efficient and practical [Aki and Richards, 2002; Yan and Sava, 2009; Han et al., 2016]. However, Sun et al. [2001] showed that they induce amplitude distortion and phase shift. Such problems can be fully avoided by using the decomposition operators [Zhang and McMechan, 2010] or the decoupled wave equations [Ma and Zhu, 2003; Li et al., 2007; Du et al., 2017]. The decomposition method can fully retain the amplitude and phase of the P- and S-waves. The decomposition operators are applied to the subsurface wavefield and are not related to the wavefield extrapolation. Therefore, the decoupled wave equations need improved extrapolation methods.

The elastic wavefield extrapolation algorithm is the core of processing such as elastic wave simulation, reverse time migration (RTM), and full waveform inversion. Those techniques require an efficient and accurate algorithm, in which the large time step is a key to the efficiency. Conventional finite-difference methods [Virieux, 1984] and pseudo-spectral methods [Reshef et al., 1988] are the two popular methods of wave extrapolation. However, conventional finite different methods are constrained to the Courant-Friedrch-Lewy (CFL) condition and require small time steps to guarantee stability [Finkelstein and Kastner, 2007]. The pseudo-spectral methods, equivalent to infinite order finite-difference, require even smaller time step. A solution to this issue lies in compensating error of approximating the temporal derivatives approximation in spatial derivative computation. For finite-differencebased methods, the optimized coefficients of finite-difference in joint time-space domain could become a good choice to improve the accuracy [Tan and Huang, 2014; Liu and Sen,2009, 2011]. For the spectral-based method, incorporation of the temporal compensation factors into the extrapolation operators in the mixed domain is a desirable way to reduce the artifacts caused by the temporal dispersion [Soubaras and Zhang,2008; Zhang and Zhang, 2009; Stoffa and Pestana, 2009; Etgen and Brandsherg-Dahl, 2009; Du et al., 2013].

The k-space method is another type of the spectral domain wave extrapolation method. It is essentially an extension of the pesudo-spectral method and reaches high accuracy by using a temporal iteration procedure in combination with the accurate spectral evolution of spatial derivatives [Tabei, et al., 2002]. The dispersion errors of the second-order time integration operators are compensated by this procedure [Fang et al., 2014]. Early attempts of the k-space method include Fornberg and Whitham [1978]'s application to nonlinear wave equations and Bojarski [1982]'s research on the scattering problem. It is then extended by many researches including the application to the elastic scalar wave equation [Compani-Tabrizi, 1986] and the application to the acoustical wave equation [Pourjavid and Tretiak, 1992]. The first-order wave equations are capable of handling velocity and density variations and are easier to apply the perfectly matched layer (PML) boundary condition. Hence, k-space method on the basis of firstorder wave equations attracts many attentions [Mast et al, 2001; Tabei et al., 2002; Fang et al., 2014].

However, due to its velocity-dependency [Etgen and Brandsberg-Dahl, 2009], solving the k-space operators in the spectral domain involves high computational cost. To reduce computational costs, the flexible-control lowrank methods established a great interest toward further investigation which contains the lowrank decomposition method [Fomel et al., 2013; Cheng et al., 2016] and the lowrank FD method [Song et al., 2013; Fang et al., 2014; Han et al., 2016]. The lowrank decomposition method generates a sparse separable decomposition of the operator matrix and balance the accuracy and efficiency through the rank. As a branch of the lowrank decomposition method, Sun et al. [2015] developed a lowrank one-step method. Cheng et al. [2016] proposed a series of approximate mixeddomain integral operators in the anisotropic media for elastic wave extrapolation. The lowrank FD method (LFD) is now widely used to design the FD scheme in relation to the spectral response in the mixed-domain. Song et al. 
[2013] proposed the LFD method for the propagation of the scalar wave. It is extended to a coupled first-order system to handle the variable density and velocity by Fang et al. [2014]. However, the current literature on the LFD method exerts their efforts to the scalar wave extrapolation based on the acoustic assumption and ignores the elastic nature of the earth. It is essential to develop a new LFD-based method to depict the structures of the subsurface from elasticity and linearity standpoints.

In this paper, our prime goal is to design a lowrank FD method for elastic wavefield extrapolation based on decoupled wave equations. The LFD-based elastic wave extrapolation is available to provide accurate P- and Swavefield vectors. Firstly, we begin with the common elastic displacement equations to derive second-order extrapolation operators in the mixed-domain and deduce first-order operators from these second-order operators. Then, for heterogeneous media, we formulate a discretization form of the first-order decoupled system. Next, the lowrank approximation is applied in the first-order extrapolation operators and the corresponding staggered-grid LFD scheme is designed. Finally, to validate the proposed method, we implement the elastic wave simulation on the simple models and on the complex ones, respectively. We also apply our proposed method to elastic RTM on the Marmousi2 model.

\section{Methodology}

\subsection{Second-order Elastic Wave Extrapolation Operators in Mixed-domain}

The LFD-based elastic wave extrapolation matters to the construction of the decoupled P- and S-wavefield. In consideration of the decoupled wave equations, we derive second-order extrapolation operators to obtain secondorder elastic wave extrapolation in the mixed-domain. According to the elastic wavefield vector decomposition method [Zhang and McMechan, 2010], we could obtain the decoupled elastic wave equations from the common second-order elastic wave equations [Aki and Richard, 2002]. Given that the velocity terms are constant, we employ the analytical solutions to these decoupled elastic wave equations and formulate the second-order extrapolation operators.

In a 2D infinite, homogeneous, and isotropic medium, the elastic wave equation is [Aki and Richards, 2002]:

$$
\frac{\partial^{2} \mathbf{u}}{\partial t^{2}}-c_{p}^{2} \nabla(\nabla \cdot \mathbf{u})+c_{s}^{2} \nabla \times(\nabla \times \mathbf{u})=0
$$

where $\mathbf{u}=\left(u_{x}, u_{z}\right)^{T}$ denotes a displacement vector, $t$ represents the time, $c_{p}$ is the P-wave particle velocity, $c_{s}$ is the Swave particle velocity, and $\mathbf{x}=(x, z)^{T}$ is the spatial location vector. Note that in this paper, the discussions and numerical tests are in 2D cases. Given that the velocity terms are constant, we could derive the following equations in the wavenumber domain:

$$
\frac{\partial^{2} \tilde{\mathbf{U}}}{\partial t^{2}}+c_{p}^{2} \mathbf{k}(\mathbf{k} \cdot \tilde{\mathbf{U}})-c_{s}^{2} \mathbf{k} \times(\mathbf{k} \times \tilde{\mathbf{U}})=0
$$

where $\mathbf{k}=\left(k_{x}, k_{z}\right)^{T}$ represents the vector of wavenumber. The vector of $\tilde{\mathbf{U}}$ represents the displacement vector in the domain of wavenumber, and the forward Fourier transform is expressed as

$$
\tilde{\mathbf{U}}=\frac{1}{(2 \pi)^{2}} \int \mathbf{u} \cdot e^{-i \mathbf{k} \cdot \mathbf{x}} \mathrm{d} \mathbf{x} .
$$

Equations (1) and (2) are the coupled elastic wave formulas in which the displacement vector contains not only the P-wave but also the S-wave. For a physical interpretation of seismic processing results (e.g., seismic imaging profiles), it is necessary to implement wavefield separation or decomposition. The derivation of decoupled wave equations is based on the condition that $\mathrm{P}$ - and $\mathrm{S}$-waves propagate independently in homogeneous media. The decomposed P- and S-wavefields [Zhang and McMechan, 2010] could be expressed as 


\section{Qizhen Du et al.}

$$
\begin{gathered}
\tilde{\mathbf{U}}_{\mathbf{p}}=\tilde{\mathbf{k}}(\tilde{\mathbf{k}} \cdot \tilde{\mathbf{U}}) \\
\tilde{\mathbf{U}}_{s}=-\tilde{\mathbf{k}} \times(\tilde{\mathbf{k}} \times \tilde{\mathbf{U}})
\end{gathered}
$$

where $\tilde{\mathbf{k}}=\frac{\mathbf{k}}{|\mathbf{k}|}$ is the unit vector of wavenumber, $\tilde{\mathbf{U}}_{\mathrm{p}}$ and $\tilde{\mathbf{U}}_{s}$ represent the P-and S-wave vectors in the wavenumber domain, respectively. Under the linear assumption, the vector wavefield is expressed as the superposition of P-wave (irrotational) part and S-wave (non-divergence) part:

$$
\tilde{\mathbf{U}}=\tilde{\mathbf{U}}_{\mathrm{p}}+\tilde{\mathbf{U}}_{s}
$$

Substituting the vector wavefield expressed by equation (6) into equation (2), and applying the identities in connection with the divergence and the curl, the decoupled wave equations can be given as

$$
\frac{\partial^{2} \tilde{\mathbf{U}}_{\mathrm{p}}}{\partial t^{2}}+c_{p}^{2} \mathbf{k}(\mathbf{k} \cdot \tilde{\mathbf{U}})=0
$$

and

$$
\frac{\partial^{2} \tilde{\mathbf{U}}_{s}}{\partial t^{2}}-c_{s}^{2} \mathbf{k} \times\left(\mathbf{k} \times \tilde{\mathbf{U}}_{s}\right)=0
$$

equations (7) and (8) characterize the P- and S-wave propagations, respectively. The derivation details are given in Appendix A.

According to the plane wave theory, analytical solutions to equations (7) and (8) are expressed as

and

$$
\tilde{\mathbf{U}}_{\mathrm{p}}=\tilde{\mathbf{k}}\left(\tilde{\mathbf{k}} \cdot \tilde{\mathbf{U}}_{0}\right) e^{ \pm i c_{p} \mathbf{k} t}
$$

$$
\tilde{\mathbf{U}}_{s}=-\tilde{\mathbf{k}} \times\left(\tilde{\mathbf{k}} \times \tilde{\mathbf{U}}_{0}\right) e^{ \pm i c_{s} \mathbf{k} t}
$$

Herein, $\tilde{\mathbf{U}}_{0}$ is the amplitude vectors. The sign \pm denotes that the solution stands for the outgoing or the ingoing wavefield.

We merge the analytical solutions into the temporal discretization equation of the leapfrog form and obtain the mixed-domain integral solutions for the elastic case as follows:

$$
\begin{gathered}
\tilde{\mathbf{U}}_{p}(t+\Delta t)+\tilde{\mathbf{k}}_{p}(t-\Delta t)-2 \tilde{\mathbf{U}}_{p}(t)=\tilde{\mathbf{k}}(\tilde{\mathbf{k}} \cdot \tilde{\mathbf{U}}(t)) \cdot\left(2\left(\cos \left(|k| c_{p} \Delta t\right)-1\right)\right) \\
\tilde{\mathbf{U}}_{s}(t+\Delta t)+\tilde{\mathbf{k}}_{s}(t-\Delta t)-2 \tilde{\mathbf{U}}_{s}(t)=-\tilde{\mathbf{k}} \times(\tilde{\mathbf{k}} \times \tilde{\mathbf{U}}(t)) \cdot\left(2\left(\cos \left(|k| c_{s} \Delta t\right)-1\right)\right)
\end{gathered}
$$

Note that the equation related to $\mathrm{P}$-wave only describes the P-wave propagation, which means that if the terms related to S-wave are added to the P-wave equation, the products of the interaction between these terms and the propagation operators are zeros. Therefore, under the condition that no effects of the S-wave exist in the P-wave formulas, we can replace the P-wavefield symbol $\tilde{\mathbf{U}}_{p}$ with the wavefield symbol $\tilde{\mathbf{U}}$ at the right-hand side of equation (11). Similarly, we can determine the S-wave propagation equation as shown by equation (12).

equations (11) and (12) are the exact discretization representations of the second-order time derivatives. When describing equations (11) and (12) at the component level, we could obtain the mixed-domain terms:

$$
\left\{\begin{array}{l}
\tilde{k}_{j} \tilde{k}_{l}\left(2\left(\cos \left(|k| c_{p} \Delta t\right)-1\right)\right) \\
\tilde{k}_{j} \tilde{k}_{l}\left(2\left(\cos \left(|k| c_{s} \Delta t\right)-1\right)\right)
\end{array}\right.
$$

Indices $j, l=1,3$ denote $x$-and $z$-axes of the Cartesian coordinate in a 2D medium and the mixed-domain terms 
are the kernels of the mixed-domain recursive time integration operators [Pestana and Stoffa, 2010; Du et al., 2013] for the elastic case. We use the Taylor expansions to approximate these exact mixed-domain operators and find that the pseudo-spectral operators, in the elastic case, are only the second-order Taylor polynomial approximations of these operators. In this sense, it is the high-order terms of the mixed-domain operators' Taylor expansion polynomials that compensate the temporal accuracy loss caused by the second-order polynomials.

\subsection{First-order Elastic Wave Extrapolation Operators in Mixed-Domain}

In practice, the wavefield extrapolation methods based on the first-order velocity-stress formulas catch higher ability to handle a variant density situation than the second-order displacement formulas [Virieux, 1984 and 1986]. It is easier to combine the PML boundary conditions [Bérenger, 1994; Yuan et al., 2014] with the first-order system of elastic wave equations [Tabei et al., 2002]. Therefore, we extend the method demonstrated above to the firstorder system.

If we define $v_{j}$ and $\tau_{j l}$ as the particle velocity and stress components, respectively, the velocity-stress formulas [Leaney, 1988] follow that:

$$
\left\{\begin{array}{c}
\frac{\partial v_{l}}{\partial t}=\frac{1}{\rho(\mathbf{x})} \frac{\partial \tau_{j l}}{\partial x_{j}} \\
\frac{\partial \tau_{j l}}{\partial x_{j}}=\lambda(\mathbf{x}) \delta_{j l} \frac{\partial v_{m}}{\partial x_{m}}+\mu(\mathbf{x})\left(\frac{\partial v_{l}}{\partial x_{j}}+\frac{\partial v_{j}}{\partial x_{l}}\right)
\end{array},\right.
$$

where $\lambda(\mathbf{x})$ and $\mu(\mathbf{x})$ are the lamé coefficients, and $\rho(\mathbf{x})$ is density. The subscripts ${ }_{j l l m}$ represent the components of the Cartesian coordinate system, and $\delta_{j l}$ is the Kronecker delta.

For homogeneous media, the formulas in the time-wavenumber domain are determined as

$$
\left\{\begin{array}{c}
\frac{\partial \tilde{v}_{l}}{\partial t}=\frac{1}{\rho} i k_{j} \tilde{\tau}_{j l} \\
\frac{\partial \tilde{\tau}_{j l}}{\partial x_{j}}=\lambda \delta_{j l} i k_{m} \tilde{v}_{m}+\mu\left(i k_{j} \tilde{v}_{l}+i k_{l} \tilde{v}_{j}\right)
\end{array},\right.
$$

where $i$ is the imaginary unit, $\lambda$ and $\mu$ are Lame's constants, and $\tilde{v}_{j}, \tilde{\tau}_{j l}$ are the 2D spatial forward Fourier transforms of particle velocity and stress components, respectively.

To derive the first-order operator in the mixed-domain, we employ the trigonometric identities $\cos (x)=1$ $2 \sin ^{2}(x / 2)$ and $\sin (x)=\operatorname{sinc}(x) / x$ to reshape operators (13), then we obtain

$$
\left\{\begin{array}{l}
i^{2} k_{j} k_{l} c_{p}^{2} \Delta t \operatorname{sinc}^{2}\left(\frac{|\mathbf{k}| c_{p} \Delta t}{2}\right) \\
i^{2} k_{j} k_{l} c_{s}^{2} \Delta t \operatorname{sinc}^{2}\left(\frac{|\mathbf{k}| c_{s} \Delta t}{2}\right)
\end{array}\right.
$$

Note that, in the equations above, replacing the pseudo spectral operator $i^{2} k_{j} k_{l}$ with $i^{2} k_{j} k_{l} c_{p, s}^{2} \Delta t^{2} \operatorname{sinc}^{2}\left(\frac{\mathbf{k} \mid c_{p, s} \Delta t}{2}\right)$ improves the accuracy of the numerical simulation. In a first-order system, the pseudo-spectral operator is changed into the following form at the large time-step without lowering the accuracy

$$
i k_{l} \operatorname{sinc}^{2}\left(\frac{|\mathbf{k}| c_{p, s} \Delta t}{2}\right) .
$$

However, the velocity and stress components in equation (14) are not only related to P-wave but also to S-wave. We introduce the variables $v_{s j}=\partial u_{j}^{p} / \partial t$ and $v_{j}^{s}=\partial u_{j}^{s} / \partial t$ as P-and S-wave particle velocity components, respectively. 


\section{Qizhen Du et al.}

These variables follow the linear principle, $v_{j}=v_{j}^{p_{j}}+v_{j}^{s}$. The second-order decoupled wave equations are transformed to the first-order ones (Li et al., 2007):

$$
\begin{aligned}
& \left\{\begin{array}{c}
\frac{\partial \tau^{p}}{\partial t}=(\lambda+2 \mu)\left(\frac{\partial v_{x}}{\partial x}+\frac{\partial v_{z}}{\partial z}\right) \\
\frac{\partial v_{x}^{p}}{\partial t}=\frac{1}{\rho} \frac{\partial \tau^{p}}{\partial x} \\
\frac{\partial v_{z}^{p}}{\partial t}=\frac{1}{\rho} \frac{\partial \tau^{p}}{\partial z}
\end{array}\right. \\
& \left\{\begin{array}{c}
\frac{\partial v_{x}^{s}}{\partial t}=\frac{1}{\rho}\left(\frac{\partial \tau_{x z}^{S}}{\partial z}+\frac{\partial \tau_{x x}^{s}}{\partial x}\right) \\
\frac{\partial v_{z}^{s}}{\partial t}=\frac{1}{\rho}\left(\frac{\partial \tau_{x z}^{S}}{\partial z}+\frac{\partial \tau_{z z}^{s}}{\partial x}\right) \\
\frac{\partial \tau_{x z}^{S}}{\partial t}=\mu\left(\frac{\partial v_{x}}{\partial z}+\frac{\partial v_{z}}{\partial x}\right) \\
\frac{\partial \tau_{x x}^{S}}{\partial t}=-2 \mu \frac{\partial v_{z}}{\partial z} \\
\frac{\partial \tau_{z z}^{S}}{\partial t}=-2 \mu \frac{\partial v_{x}}{\partial x}
\end{array}\right.
\end{aligned}
$$

where $\tau^{p}$ is $\mathrm{P}$-wave stress, $\tau_{x x}^{s}$ and $\tau_{z z}^{s}$ are $\mathrm{S}$-wave normal stresses and $\tau^{s}{ }_{x z}$ is $\mathrm{S}$-wave shear stress.

We use the forward 2D spatial Fourier transform to derive the representations in the wavenumber domain

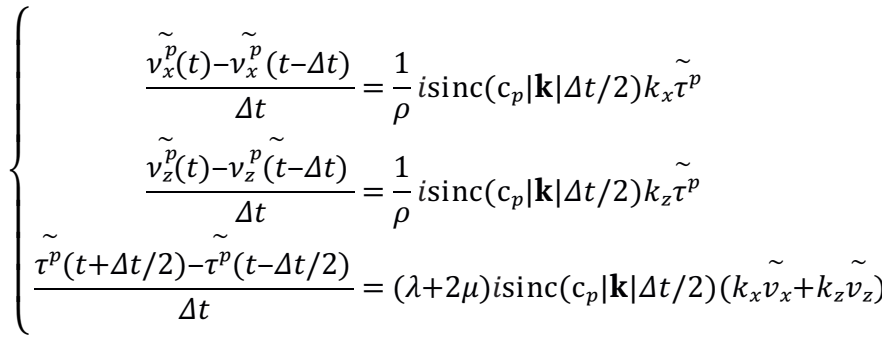

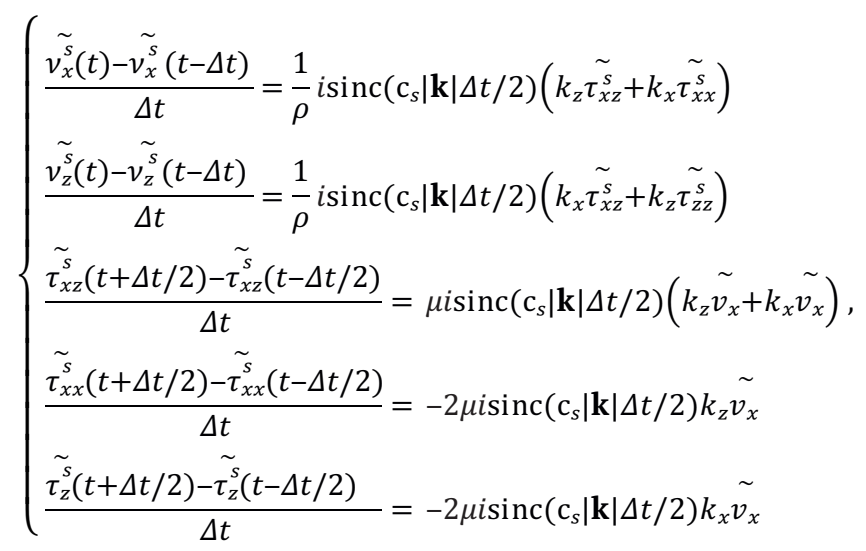

where $\tilde{v}_{j}^{p}, \tilde{v}_{j}{ }_{j}, \tilde{\tau}^{p}$ and $\tilde{\tau}_{j l}^{s}$ are the Fourier transform of , $v_{j}^{p}, v_{j}^{s}, \tau^{p}$ and $\tau^{s}{ }_{j l}$. After that, we will design the decoupled first-order system of the elastic wave on staggered grids.

Figure 1 describes a schematic of the 2D staggered grid scheme. We use $\Delta x$ and $\Delta z$ to denote the space grid intervals along the $\mathrm{x}$ - and $\mathrm{z}$-axes, respectively, and $\mathrm{i}$ and $\mathrm{j}$ to denote the grid cell indices, respectively. $\tau_{p}, \tau_{x x}^{s}$ and $\tau_{z z}^{s}$ are discretized at space locations $(i \Delta x, j \Delta z), \tau_{x z}^{s}$ is discretized at space locations, $((i+0.5) \Delta x,(j+0.5) \Delta z) v_{x}^{p}$ and $v_{x}^{s}$ are discretized at space locations $((i+0.5) \Delta x, j \Delta z)$, and $v_{z}{ }_{z}$ and $v_{z}^{s}$ are discretized at space locations $(i \Delta x,(j+0.5) \Delta z)$. In the discussion, the staggered-grid schemes are used for theoretical analyses and numerical tests. 
In the wavenumber domain, the staggered concepts conveyed by Figure 1 are achieved with the shift properties of Fourier transform. When the gradient of velocity is small, we can replace constant velocities, $c_{p}$ and $c_{s}$, with the space-dependent velocities, $c_{p}(\mathbf{x})$ and $c_{s}(\mathbf{x})$ [Fang, et al., 2014]. Applying reverse Fourier transforms to equations (20) and (21) yields:

$$
\begin{aligned}
& \left\{\begin{array}{l}
v_{x}=v_{x+v_{x}^{s}}^{p} \\
v_{z}=v_{z}^{p} v_{z}^{s}
\end{array}\right. \\
& \left\{\begin{array}{l}
\frac{v_{x}^{p}(t+\Delta t / 2)-v_{x}^{p}(t-\Delta t / 2)}{\Delta t}=\frac{1}{\rho(\mathbf{x})} \frac{\partial \tau \rho}{\partial x^{+}} \\
\frac{v_{z}^{p}(t+\Delta t / 2)-v_{z}^{p}(t-\Delta t / 2)}{\Delta t}=\frac{1}{\rho(\mathbf{x})} \frac{\partial \tau \rho}{\partial z^{+}} \\
\frac{\tau^{p}(t+\Delta t / 2)-\tau^{p}(t-\Delta t / 2)}{\Delta t}=(\lambda(\mathbf{x})+2 \mu(\mathbf{x}))\left(\frac{\partial_{p} v_{x}}{\partial x^{-}}+\frac{\partial_{p} v_{z}}{\partial z^{-}}\right)
\end{array}\right. \\
& \left\{\begin{array}{l}
\frac{v_{x}^{s}(t+\Delta t / 2)-v_{x}^{s}(t-\Delta t / 2)}{\Delta t}=\frac{1}{\rho(\mathbf{x})}\left(\frac{\partial_{s} \tau_{x z}^{s}}{\partial z^{-}}+\frac{\partial \tau_{x x}^{s}}{\partial x^{-}}\right) \\
\frac{v_{z}^{s}(t+\Delta t / 2)-v_{z}^{s}(t-\Delta t / 2)}{\Delta t}=\frac{1}{\rho(\mathbf{x})}\left(\frac{\partial_{s} \tau_{x z}^{s}}{\partial x^{+}}+\frac{\partial_{s} \tau_{z z}^{s}}{\partial z^{+}}\right) \\
\frac{\tau_{x x}^{s}(t+\Delta t / 2)-\tau_{x x}^{s}(t-\Delta t / 2)}{\Delta t}=-2 \mu(\mathbf{x}) \frac{\partial_{s} v_{z}}{\partial z^{-}} \\
\frac{\tau_{z z}^{s}(t+\Delta t / 2)-\tau_{z z}^{s}(t-\Delta t / 2)}{\Delta t}=-2 \mu(\mathbf{x}) \frac{\partial_{s} v_{x}}{\partial x^{-}} \\
\frac{\tau_{x z}^{s}(t+\Delta t / 2)-\tau_{x z}^{s}(t-\Delta t / 2)}{\Delta t}=\mu(\mathbf{x})\left(\frac{\partial_{s} v_{x}}{\partial z^{+}}+\frac{\partial v_{z}}{\partial x^{+}}\right)
\end{array}\right.
\end{aligned}
$$

where

$$
\left\{\begin{array}{l}
\frac{\partial_{p}}{\partial x_{j}^{+}}=F^{-1}\left\{i k_{j} e^{+i k_{j} \Delta_{j} / 2} \operatorname{sinc}\left(\mathrm{c}_{p}(\mathbf{x})|\mathbf{k}| \Delta t / 2\right) F\{\cdot\}\right\} \\
\frac{\partial_{p}}{\partial x_{j}^{-}}=F^{-1}\left\{i k_{j} e^{-i k_{j} \Delta_{j} / 2} \operatorname{sinc}\left(\mathrm{c}_{p}(\mathbf{x})|\mathbf{k}| \Delta t / 2\right) F\{\cdot\}\right\} \\
\frac{\partial_{s}}{\partial x_{j}^{+}}=F^{-1}\left\{i k_{j} e^{+i k_{j} \Delta_{j} / 2} \operatorname{sinc}\left(\mathrm{c}_{p}(\mathbf{x})|\mathbf{k}| \Delta t / 2\right) F\{\cdot\}\right\} \\
\frac{\partial_{s}}{\partial x_{j}^{-}}=F^{-1}\left\{i k_{j} e^{-i k_{j} \Delta_{j} / 2} \operatorname{sinc}\left(\mathrm{c}_{p}(\mathbf{x})|\mathbf{k}| \Delta t / 2\right) F\{\cdot\}\right\}
\end{array}\right.
$$

These derivative operators are named as the first-order k-space operators [Tabei, 2002]. Note that equations (23) are actually the approximations of the exact ones which use constant velocities in the sinc operators. Its accuracy is still relatively high (Fang et al., 2014] in media with small velocity gradient. And it also allows us to testify this method in the complex model with large velocity gradient.

For numerical solutions to the spatial derivatives in equation (22), we could introduce the localized Fourier transform methods [Wards, 2008; Etgen and Brandsberg-Dhl, 2009; Zhang and Zhang, 2009]. However, these methods have access to all nodes in the mixed-domain and require several multidimensional inverse and forward Fourier transforms. Let $N_{x}$ stand for the total number of the spatial grids, the computational complexity of the localized Fourier transform methods amounts to $O\left(N_{x}^{2}\right)$. From the mathematical perspective, the mixed-domain operators usually exhibit an oscillatory behavior and approximate lowrank [Candes et al., 2007; Engquist and Ying, 2009]. Therefore, one choice for reducing the computational complexity is the application of the lowrank methods [Fomel et al., 2013; Fang et al., 2014]. In the next section, we will demonstrate the details of the lowrank approximation scheme and the lowrank finite-difference method. 

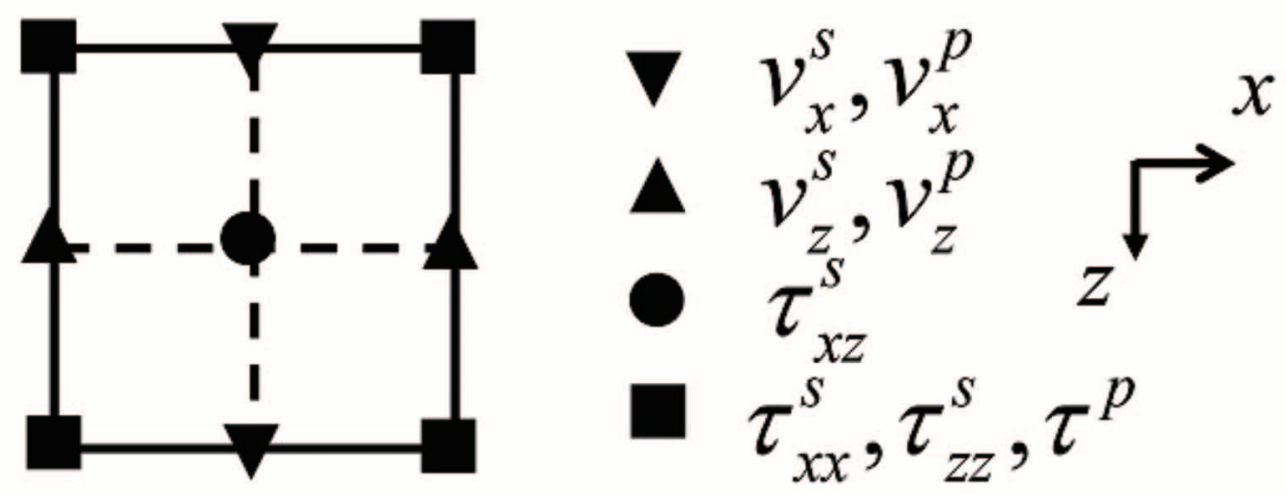

Figure 1. A schematic of a 2D staggered grid scheme.

\subsection{Lowrank Approximation to First-order operators in the Heterogeneous Case}

It is not desirable to extrapolate the elastic wave using the recursive time integration operators because of the high computational cost. In this section, we will derive the decomposition representations of the mixed-domain operators with the lowrank decomposition method [Fomel et al., 2013; Cheng et al., 2016]. As equation (23) expressed, the mixed-domain operators can be categorized into two groups. One is associated with $\mathrm{P}$-wave and the other is associated with $S$-wave. For a brief description, we will take $\frac{\partial_{p} v_{x}}{\partial x_{j}^{+}}$as an example to introduce the approximation. Let $W_{j}^{p}(\mathbf{x}, \mathbf{k})=k_{j} \operatorname{sinc}\left(c_{p}(\mathbf{x})|\mathbf{k}| \Delta t / 2\right)$ which is an $N_{x}$ by $N_{x}$ matrix, $\mathbf{x}=\left\{x_{1}, \ldots, x_{N_{x}}\right\}$ represent samples in the space domain and $\mathbf{k}=\left\{k_{1}, \ldots, k_{N_{x}}\right\}$ represent samples in the wavenmber domain. The exact operator, $W_{j}^{p}(\mathbf{x}, \mathbf{k})$, could be factorized into the product of three submatrices, which has the form as

$$
W_{j}^{p}(\mathbf{x}, \mathbf{k}) \approx \sum_{m=1}^{M} \sum_{n=1}^{N} W_{j}^{p}\left(\mathbf{x}, \mathbf{k}_{m}\right) a_{m n} W_{j}^{p}\left(\mathbf{x}_{n}, \mathbf{k}\right)
$$

where $W_{j}^{p}\left(\mathbf{x}, \mathbf{k}_{m}\right)$ is a submatrix of the original matrix $W_{j}^{p}(\mathbf{x}, \mathbf{k})$ consisting of M columns selected from $W_{j}^{p}(\mathbf{x}, \mathbf{k})$ related to $\mathbf{k}_{m}$, while $W_{j}^{p}\left(\mathbf{x}_{n}, \mathbf{k}\right)$ is another submatrix of the original matrix $W_{j}^{p}(\mathbf{x}, \mathbf{k})$ consisting of N rows selected from $W_{j}^{p}(\mathbf{x}, \mathbf{k})$ related to $\mathbf{x}_{n} \cdot a_{m n}$ represents the middle coefficients which have M-by-N entries, and $\mathrm{M}$ and $\mathrm{N}$ stand for the rank of the decomposition. The way to construct the decomposition representations follows the method by Engquist and Ying [2007, 2009]. The computational complexity of the algorithm is $O\left(N N_{x} \log N_{x}\right)$, and the computational cost of the Fourier transform is $N_{x} \log N_{x}$. M and $\mathrm{N}$ are usually far less than the spatial number, $N_{x}$. The ranks are up to the complexity of the model. For homogeneous media, the mixed-domain operators are equivalent to the pseudo spectral operators. There is an actual trade-off in the selection of ranks: larger values lead to a more accurate wave representation but require a longer computational time. In Fomel et al. [2013], the authors select the ranks based on an estimate of the approximation accuracy of 10-4. For the isotropic heterogeneous media, ranks between 2 and 4 can lead to satisfying accuracy [Fang et al. 2014]. In this paper, we recommend $M, N=4$. If we utilize equation (24), the approximate form of $\frac{\partial_{p} v_{x}}{\partial x_{j}^{+}}$could be given by

$$
\frac{\partial_{p} v_{x}}{\partial x_{j}^{+}} \approx \sum_{l=1}^{L} \sum_{l=1}^{L} W_{j}^{p}\left(x, k_{m}\right) a_{m n} F^{-1}\left[i e^{i k_{x} \Delta x / 2} W_{j}^{p}\left(\mathbf{x}_{n}, \mathbf{k}\right) F\left[v_{x}(\mathbf{x}, t)\right]\right]
$$

\subsection{Lowrank Finite-difference Schemes on Staggered Grids for the Elastic Case}

In this section, we will further decompose the recursive time integration operators and generate optimal coefficients for elastic wave extrapolation. The submatrix $W_{j}^{p}\left(\mathbf{x}_{n}, \mathbf{k}\right)$ is only related to the wavenumber and exhibits the behavior of oscillation [Engquist and Ying, 2009]. Thus, we could adopt Fourier sine series to represent this 
submatrix and obtain the staggered-grid lowrank finite-difference. We further decompose the third term on the right-hand side of equation (25) into:

$$
W_{j}^{p}\left(\mathbf{x}_{n}, \mathbf{k}\right) \approx \sum_{l=1}^{L} C_{j}^{p}\left(\mathbf{x}_{n}, l\right) B(l, \mathbf{k})
$$

where $B(l, \mathbf{k})$ is an $L$ by $N_{x}$ submatrix and determined as $\sin \left(\frac{2 l-1}{2} k_{j} \Delta x_{j}\right), L$ is the half size of the staggered grid stencil, $C_{j}^{p}\left(\mathbf{x}_{n}, l\right)$ is an $N$ by $L$ submatrix calculated as the product of $W_{j}^{p}\left(\mathbf{x}_{n}, \mathbf{k}\right)$ and the pseudo-inverse of $B(l, \mathbf{k})$. We introduce the auxiliary matrix

$$
G_{j}^{p}(\mathbf{x}, l)=\sum_{m=1}^{M} \sum_{n=1}^{N} W_{j}^{p}\left(\mathbf{x}, \mathbf{k}_{m}\right) a_{m n} C_{j}^{p}\left(\mathbf{x}_{n}, l\right)
$$

After that, we could overwrite the spatial derivative of $v_{x}$ as

$$
\begin{aligned}
\frac{\partial_{p} v_{x}}{\partial x_{j}^{+}} & \approx \frac{1}{2} \sum_{l=1}^{L} G_{j}^{p}(\mathbf{x}, l) F^{-1}\left[i\left(e^{i k_{j} \Delta x_{j} / 2} \mathbf{B}(l, \mathbf{k})\right) F\left(v_{x}\right)\right] \\
& \approx \sum_{l=1}^{L} G_{j}^{p}(\mathbf{x}, l) F^{-1}\left[i\left(e^{i l k_{j} \Delta x_{j}}-e^{-i(l-1) k_{j} \Delta x_{j}}\right) F\left(v_{x}\right)\right] . \\
& \approx \frac{1}{2} \sum_{l=1}^{L} G_{j}^{p}(\mathbf{x}, l) F^{-1}\left[v_{x}\left(x_{j}+l\right)-v_{x}\left(x_{j}-(l-1)\right]\right.
\end{aligned}
$$

equation (28) is analogous to the one expressed by the standard staggered grid scheme. It is a striking feature of the method that the deduction of the coefficients containing lowrank decomposition and the coefficients depend upon the media. This method could be entitled as an elastic staggered-grid lowrank FD(ESGLFD) method. The coefficients for the different media are supposed to be stored before the implementation of elastic wave simulation because of the velocity-dependency. Through the same processing, the other spatial derivatives of $v_{l}$, containing $\partial_{p} v_{x} / \partial x_{j}$, $\partial_{s} v_{x} / \partial x_{j}^{+}, \partial_{s} v_{x} / \partial x_{j}^{-}$, could be deduced as

$$
\begin{aligned}
& \frac{\partial_{p} v_{x}}{\partial x_{j}^{-}} \approx \frac{1}{2} \sum_{l=1}^{L} G_{j}^{p}(\mathbf{x}, l) F^{-1}\left[v_{x}\left(x_{j}+l-1\right)-v_{x}\left(x_{j}-l\right)\right] \\
& \frac{\partial_{s} v_{x}}{\partial x_{j}^{+}} \approx \frac{1}{2} \sum_{l=1}^{L} G_{j}^{p}(\mathbf{x}, l) F^{-1}\left[v_{x}\left(x_{j}+l\right)-v_{x}\left(x_{j}-l+1\right)\right] \\
& \frac{\partial_{s} v_{x}}{\partial x_{j}^{-}} \approx \frac{1}{2} \sum_{l=1}^{L} G_{j}^{p}(\mathbf{x}, l) F^{-1}\left[v_{x}\left(x_{j}+l-1\right)-v_{x}\left(x_{j}-l\right)\right] .
\end{aligned}
$$

Herein, we use a 2D simple homogeneous media to analyze the accuracy of the ESGLFD method. Also, we choose the spatial derivative operator, $\partial_{s} v_{x} / \partial x_{j}^{-}$as an example. The spatial derivative operator, $\partial_{p} v_{x} / \partial x_{j}^{-}$shown in equation (22), is calculated by the k-space method [Tabei et al., 2002] and the ESGLFD method, respectively. The velocity is given by $f(x, z)=20 x+25 z+1200$. The velocity value increases to $3900 \mathrm{~m} / \mathrm{s}$ (Figure $2 \mathrm{a}$ ).

Figure $2 \mathrm{~b}, 2 \mathrm{c}$ and $2 \mathrm{~d}$ illustrate the exact matrix with the $k$-space method, the approximate matrix with the ESGLFD method and the absolute error between these two matrices, respectively. There is almost no difference between Figures $2 \mathrm{~b}$ and $2 \mathrm{c}$ at the macroscopic level. By inspection, the absolute error, shown as Figure $2 \mathrm{~d}$, is almost constrained on the interval $(-0.04,0.04)$. The error is tolerant in the processing of elastic wave extrapolation.

Compared to the existing works, the elastic lowrank finite-difference operators are optimal finite-difference operators and constructed in accordance with the spectral response in certain range of frequency. The elastic recursive time integration operator of the proposed method depends on the parameters of the elastic media and the simulation which can efficiently compensate the temporal accuracy loss. It extends the application of lowrank finite-difference schemes from acoustic case to elastic situation, which provides a more accurate approximation in both temporal and spatial domains. 
Qizhen Du et al.
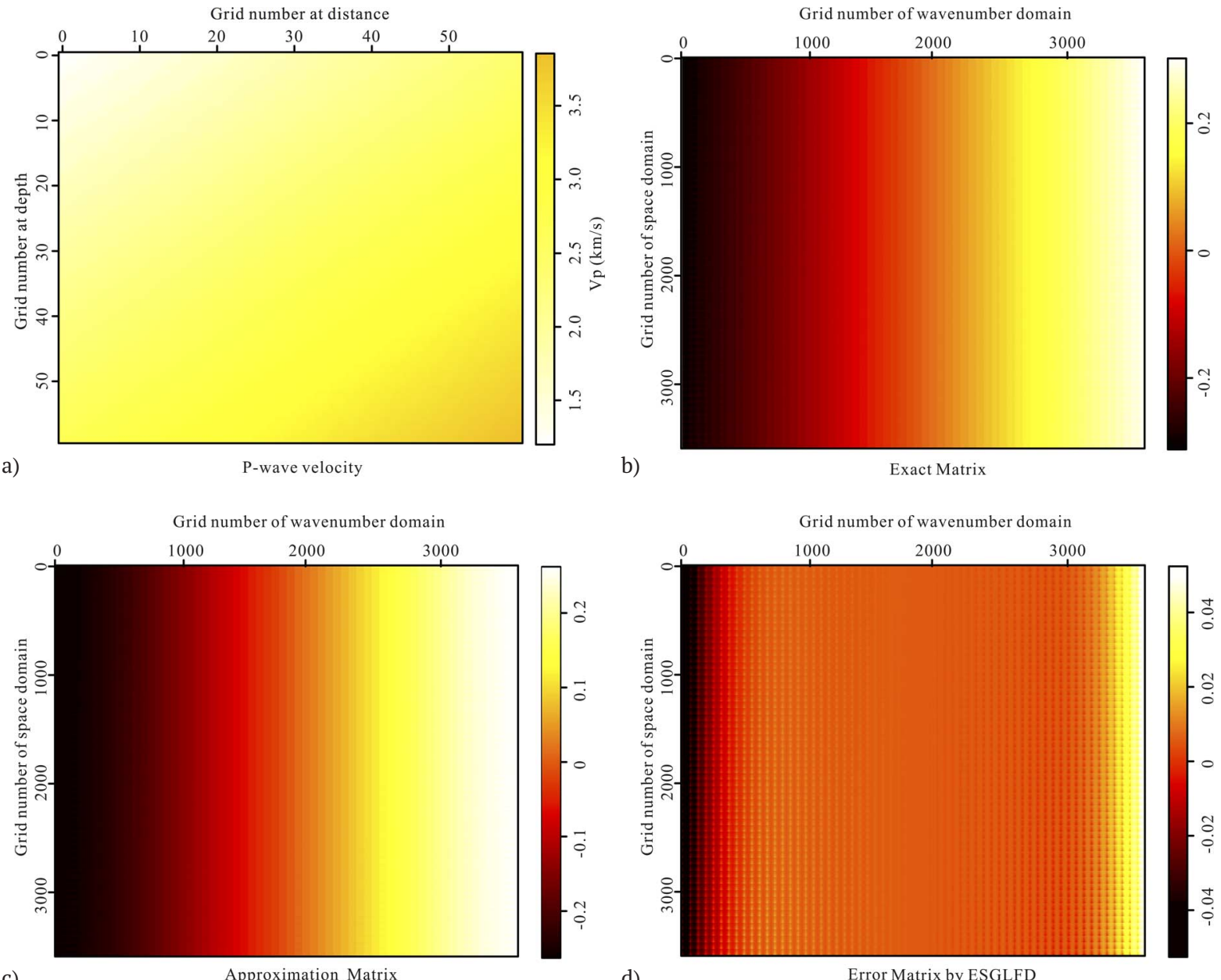

c)

Approximation Matrix

d)

Error Matrix by ESGLFD

Figure 2. (a) A linearly increasing P-wave velocity model, (b) An exact matrix for $\partial_{p} / \partial_{x j}{ }^{+}$with the k-space method, (c) An approximation matrix for $\partial_{p} / \partial_{x j}{ }^{\dagger}$ with the ESGLFD method, (d) The absolute error between the exact matrix and the approximate matrix.

\subsection{Dispersion Analysis}

We analyze the accuracy of the ESGLFD method. According to the plane wave theory, we could acquire a set of ratios containing the ratio of the $\mathrm{P}$-wave numerical velocity to the real P-wave velocity, $\xi_{p}$, and that of the $\mathrm{S}$-wave, $\xi_{s}$. These ratios indicate the errors:

$$
\xi_{p}=\frac{2 \arcsin \left(\sqrt{\left.\left(\eta_{p x}^{2}+\eta_{p z}^{2}\right) v_{p}^{2} \Delta t^{2} / 4\right)}\right.}{k \Delta t v_{p}}
$$

and

$$
\xi_{s}=\frac{2 \arcsin \left(\sqrt{\left.\left(\eta_{s x}^{2}+\eta_{s z}^{2}\right) v_{s}^{2} \Delta t^{2} / 4\right)}\right.}{k \Delta t v_{s}},
$$

where,

$$
\begin{aligned}
& \eta_{s x}=\sum_{l=1}^{L} G_{x}^{s}(\mathbf{x}, l) \sin \left((2 l-1) k_{x} \Delta h / 2\right), \\
& \eta_{s z}=\sum_{l=1}^{L} G_{z}^{s}(\mathbf{x}, l) \sin \left((2 l-1) k_{z} \Delta h / 2\right),
\end{aligned}
$$


and

$$
\begin{aligned}
& \eta_{p x}=\sum_{l=1}^{L} G_{x}{ }^{p}(\mathbf{x}, l) \sin \left((2 l-1) k_{x} \Delta h / 2\right), \\
& \eta_{p z}=\sum_{l=1}^{L} G_{z}^{p}(\mathbf{x}, l) \sin \left((2 l-1) k_{z} \Delta h / 2\right) .
\end{aligned}
$$

When $\xi=1$, no dispersion exists. If $\xi$ keeps a long way off 1 , serious dispersion will occur. Also, it is clear that the error depends on the propagation angle. Suppose that the propagation angle is $\theta$ in a 2D case, let $k_{x}=k$ $\cos (\theta)$ and $k_{z}=k \sin (\theta)$. Due to the symmetry of equations (32) and (33), we only investigate the properties of the error when $\theta \in[0, \pi / 4]$. Thereafter, we will compare the difference between the staggered grid finitedifference (SGFD) scheme and the ESGLFD scheme in terms of difference orders, velocity, time intervals and propagation angles.

Figure 3 depicts the relation between $\xi$ and $k h$ for different orders of SGFD and ESGLFD methods. Clearly, when $L$ increases, the dispersion error decreases for both SGFD and ESGLFD methods. However, the proposed ESGLFD method covers a wider wavenumber range than the SGFD method. The dispersion curves are given in Figure 4. Note that the errors change significantly with the velocities when employing the SGFD method. By comparison, the errors equal or approximately equal the limit value on the interval $[0,2.2]$ when the ESGLFD method is used. The ESGLFD method keeps highly accurate on the wavenumber range mentioned above.
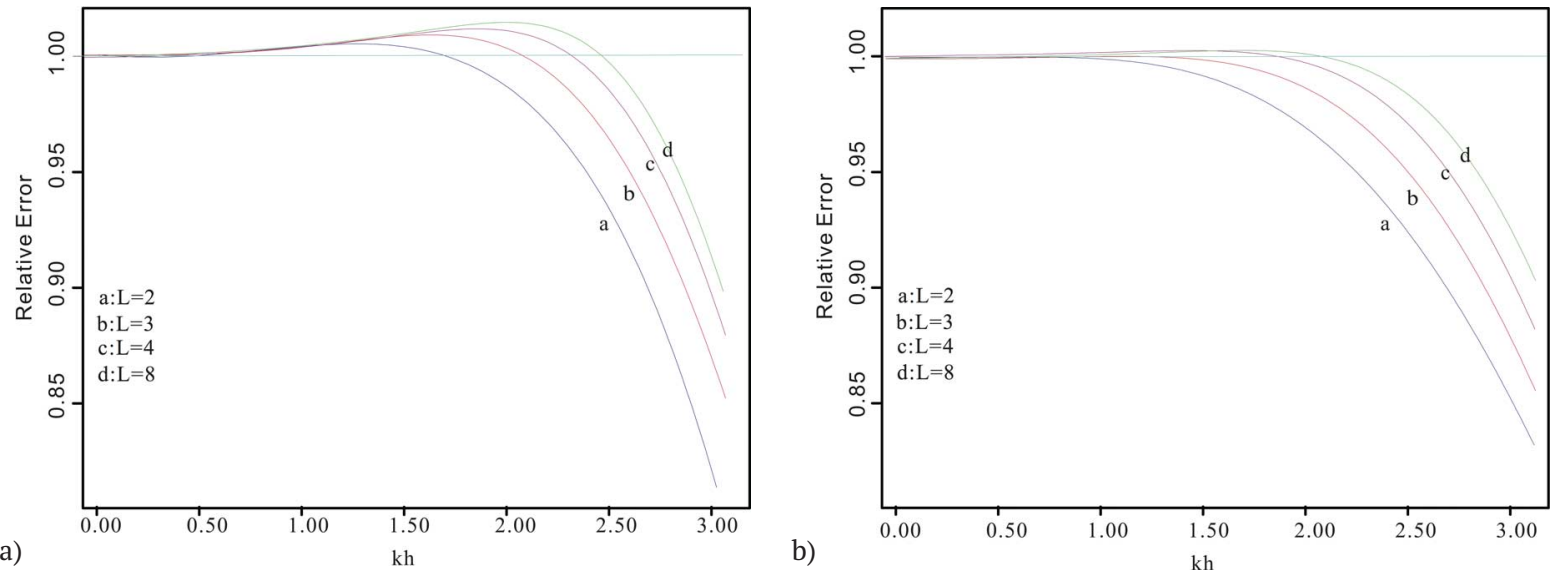

Figure 3. 2D dispersion curves of the FD methods (a) and the ESGLFD methods (b) for different orders, fourth-order ( $L=$ 2, blue), six-order ( $L=3$, red), eight-order ( $L=4$, purple), sixteen-order ( $L=8$, green), $v=3000 \mathrm{~m} / \mathrm{s}, \mathrm{D} t=1 \mathrm{~ms}$ and $\theta=\pi / 8$.

From Figure 5, we can estimate the impact of time intervals on the dispersion. The dispersion error by the SGFD method becomes larger when the time interval increases. However, as shown in Figure $5 b$, all the dispersion errors are confined in a small neighborhood of 1 with $k h \in[0,2.0]$. It proves that the ESGLFD method is effective for large time-step simulation. As mentioned above, we only investigate the dispersion relation with $\theta \in[0, \pi / 4]$ and select the representative angles including $\pi / 16, \pi / 8, \pi / 6$ and $\pi / 4$ to illustrate the variation of the dispersion errors. From Figures $6 \mathrm{a}$ and $6 \mathrm{~b}$ we can see that the trend of the dispersion curves by the two methods for the designed propagation angle is comparable. It is the wider coverage of wavenumber that gives the advantage of the ESGLFD method over the SGFD method. After the analysis of these Figures, we can find that the ESGLFD method exhibits higher accuracy. Especially, the ESGLFD method is able to yield the promise results when the time intervals become larger. 
Qizhen Du et al.

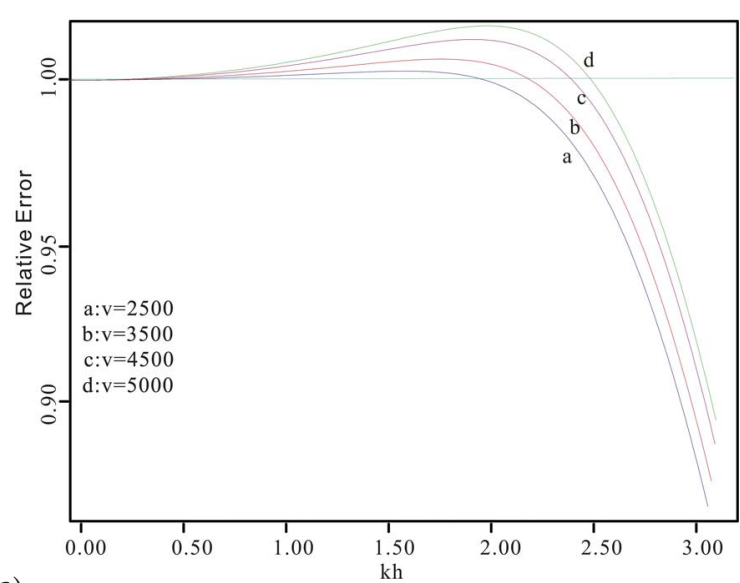

a)

Figure 4. 2D dispersion curves of the FD methods (a) and the ESGLFD methods (b) for different velocities, $v=2500 \mathrm{~m} / \mathrm{s}$ (blue), $v=3500 \mathrm{~m} / \mathrm{s}$ (red), $v=4500 \mathrm{~m} / \mathrm{s}$ (purple), $v=5000 \mathrm{~m} / \mathrm{s}$ (green), $L=6, \mathrm{D} t=1 \mathrm{~ms}$ and $\theta=\pi / 8$. a)

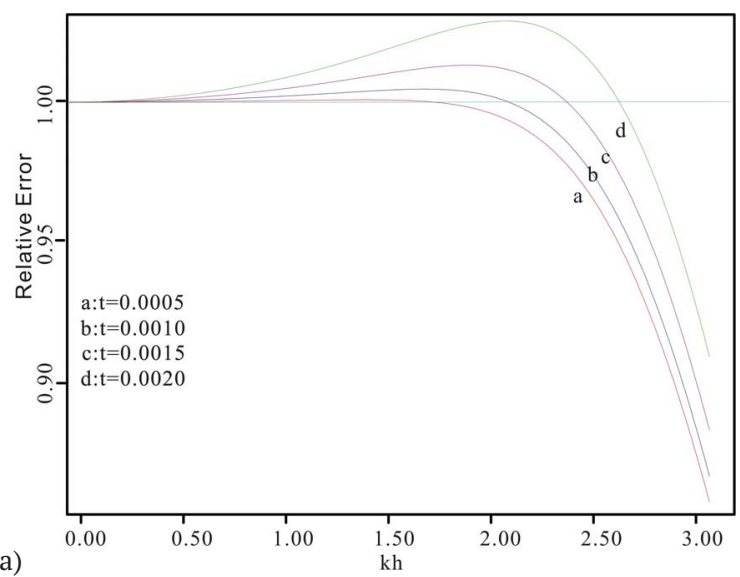

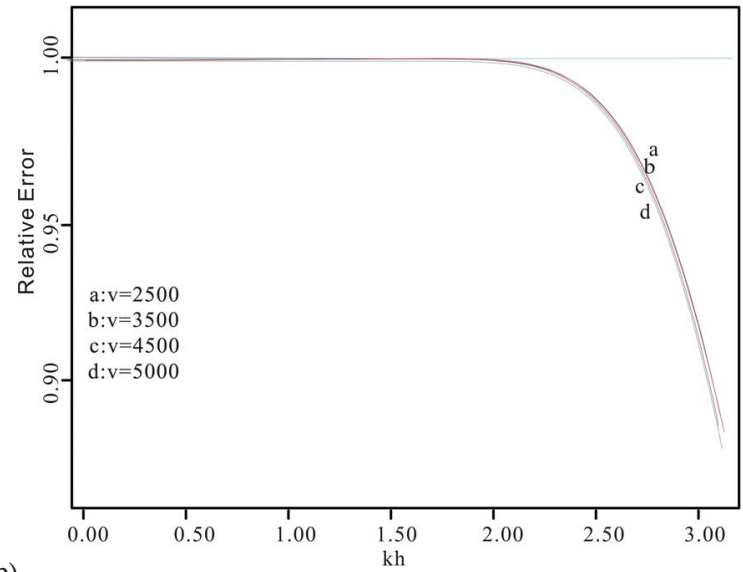

b)

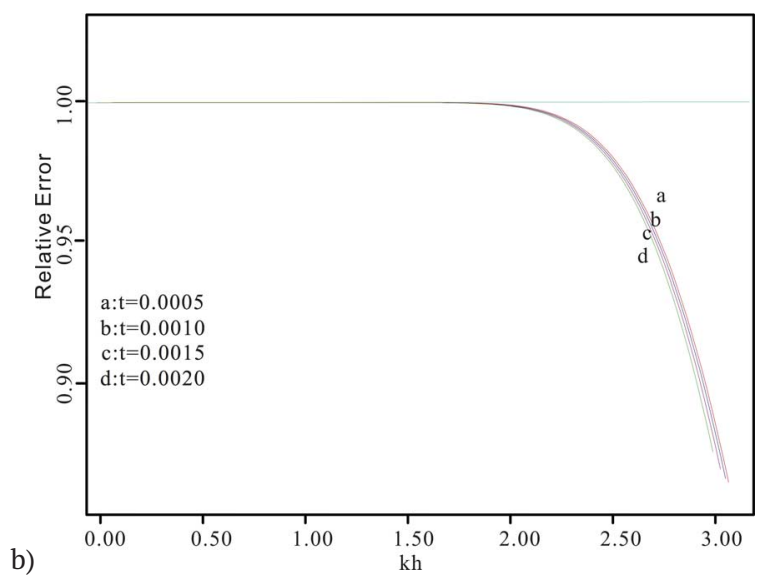

Figure 5. 2D dispersion curves of the FD methods (a) and the ESGLFD methods (b) for different time intervals, $\mathrm{D} t=0.5 \mathrm{~ms}$ (red), $\mathrm{D} t=1.0 \mathrm{~ms}$ (blue), $\mathrm{D} t=1.5 \mathrm{~ms}$ (purple), $\mathrm{D} t=2.0 \mathrm{~ms}$ (green), $v=3000 \mathrm{~m} / \mathrm{s}, L=6$ and $\theta=\pi / 8$.

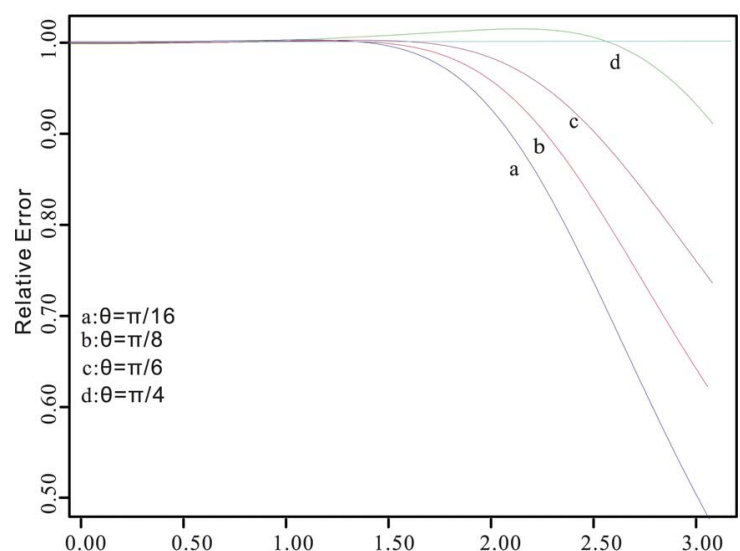

a)

Figure 6. 2D dispersion curves of the FD methods (a) and the ESGLFD methods (b) for different propagation angles, $\theta=$ $\pi / 16$ (blue), $\theta=\pi / 8$ (red), $\theta=\pi / 6$ (purple), 4 (green), $\mathrm{D} t=1 \mathrm{~ms}, v=3000 \mathrm{~m} / \mathrm{s}$ and $L=6$. 


\section{Numerical examples}

\subsection{Two-Layers Model}

To investigate the validity of the ESGLFD method, we implement an experiment on a two-layer model (Figure 7). Size of the model is defined as, with horizontal and vertical sampling intervals equal $15 \mathrm{~m}$. The time sampling interval is $2.0 \mathrm{~ms}$. The contrast of the model appears at the depth of $4500 \mathrm{~m}$. The density, P-and S-wave velocities of the upper layer are set as $2.0 \mathrm{~g} / \mathrm{cm}^{3}, 1700 \mathrm{~m} / \mathrm{s}$ and $1000 \mathrm{~m} / \mathrm{s}$, while the parameters of the lower layer are $2.2 \mathrm{~g} / \mathrm{cm}^{3}, 2550 \mathrm{~m} / \mathrm{s}$ and $1500 \mathrm{~m} / \mathrm{s}$, respectively. A Ricker wavelet is utilized as an explosive source at the location (4500m, $3975 \mathrm{~m})$, with $35 \mathrm{~Hz}$ the dominant frequency.

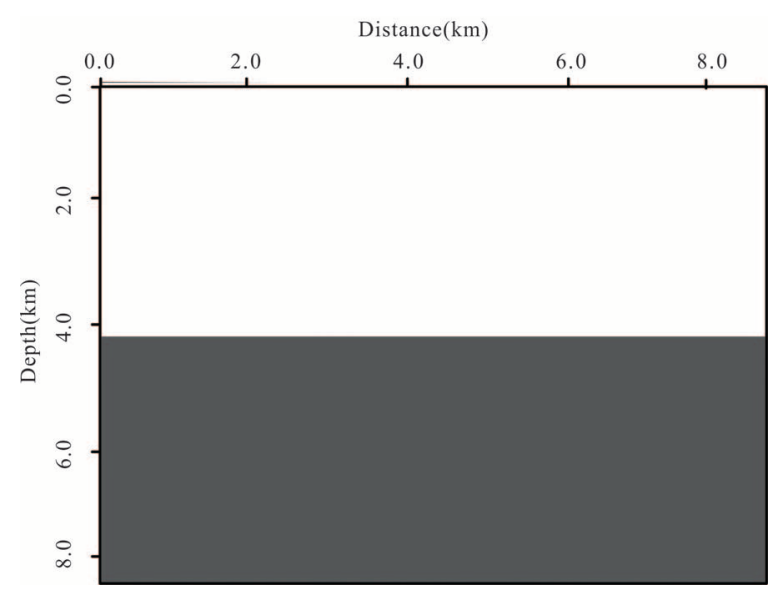

Figure 7. The geometry and the elastic parameters of a two-layer model. The pentagram denotes the excitation position for elastic wave simulation.

The $\mathrm{x}$-component snapshots for P-and S-waves at $0.8 \mathrm{~s}$ are shown in Figure 8. Visible and apparent dispersion occurs in Figures 8a and 8b. When time and space intervals become larger, it is obvious that the waveform simulated by the SGFD method is distorted. The ESGLFD method performs well and yields nearly dispersion-free results.

Figure 9 displays the vertical slices extracted from the x-component snapshots shown in Figure 8. Notice that there are three waveforms-in Figures $9 \mathrm{a}$ and $9 \mathrm{~b}$ and two waveforms in Figures $9 \mathrm{c}$ and $9 \mathrm{~d}$. It is in accordance with the fact that the reflected $\mathrm{P}$-wave, the transmission $\mathrm{P}$-wave, and the direct $\mathrm{P}$-wave exist in the snapshots, as is shown in Figures $8 \mathrm{a}$ and $8 \mathrm{~b}$, while the reflected converted S-wave and the transmission converted S-wave exist in the snapshots of Figures $8 \mathrm{c}$ and $8 \mathrm{~d}$. Meanwhile, the results also demonstrate that the wave modes, which occur in the elastic media, could be depicted by the proposed ESGLFD method effectively. The waveforms obtained by the SGFD method do not preserve the wavelet shapes well. Vibration curves occur in the neighborhood of the Ricker-shaped waveforms. Comparatively, the waveforms generated by the ESGLFD method do comply with the Ricker-wavelet forms. The results prove that the ESGLFD method is accurate and effective for elastic wave simulation when the time interval becomes larger.

\subsection{The Marmousi2 Model}

To validate our proposed method at the complex model, we select the modified elastic Marmousi2 model [Martin et al., 2006], and set the grid size of the model as $1700 \times 350$. The grid interval is redefined as $10 \mathrm{~m}$. We divide the resampled $\mathrm{P}$-wave velocity by $\sqrt{ } 3$ to calculate the $\mathrm{S}$-wave velocity. For the elastic wave simulation and the subsequent RTM, we smooth the velocity model. And Figure 10a and 10b describe the original and smoothed P-wave velocities, respectively.

In Figure 10b, subset of the elastic Marmousi2 model denoted by the dashed rectangle is used for elastic wave simulation in this experiment. The four corners are all located at the border of the resampled Marmousi 2 model, the 
Qizhen Du et al.

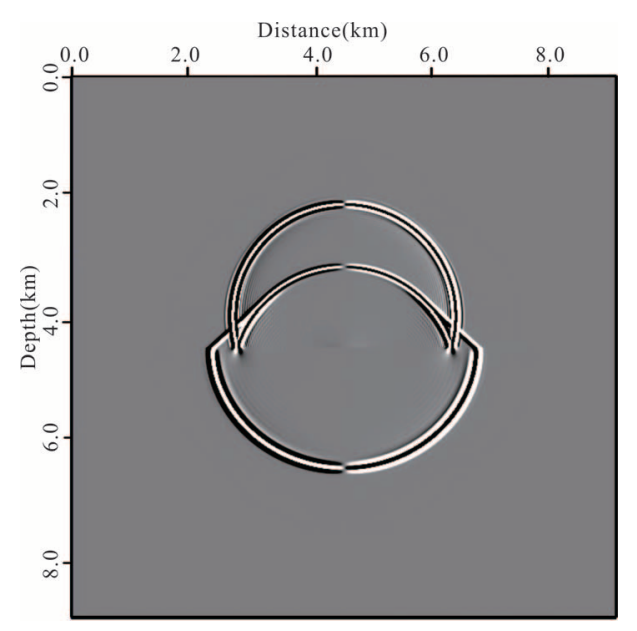

a)

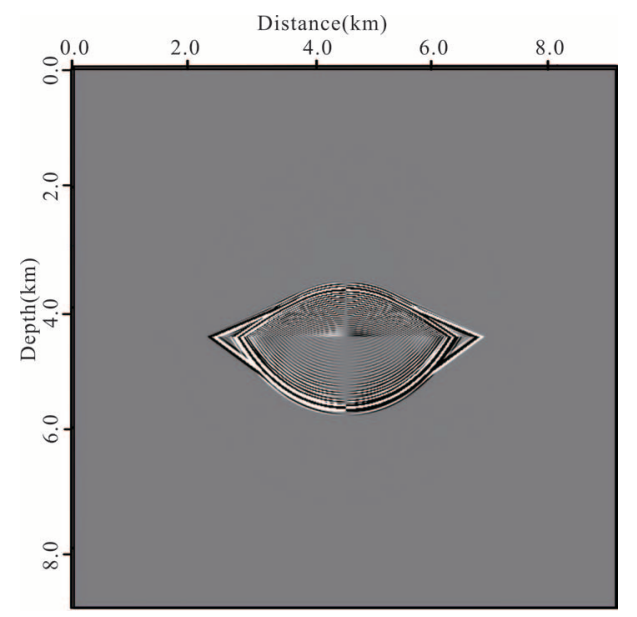

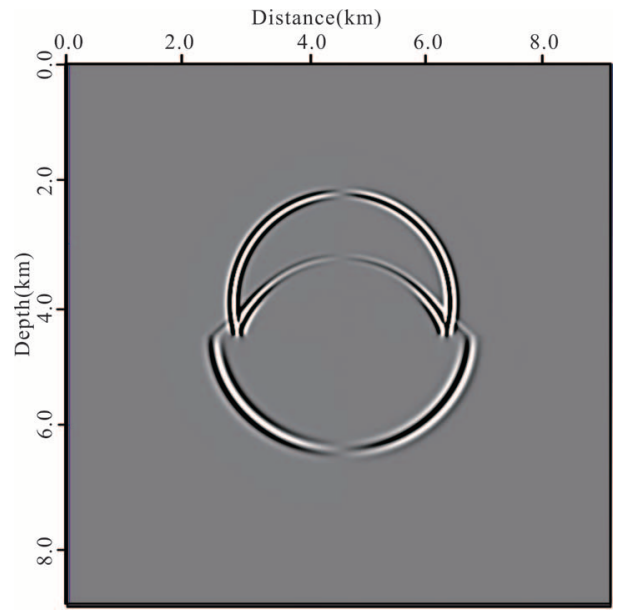

b)

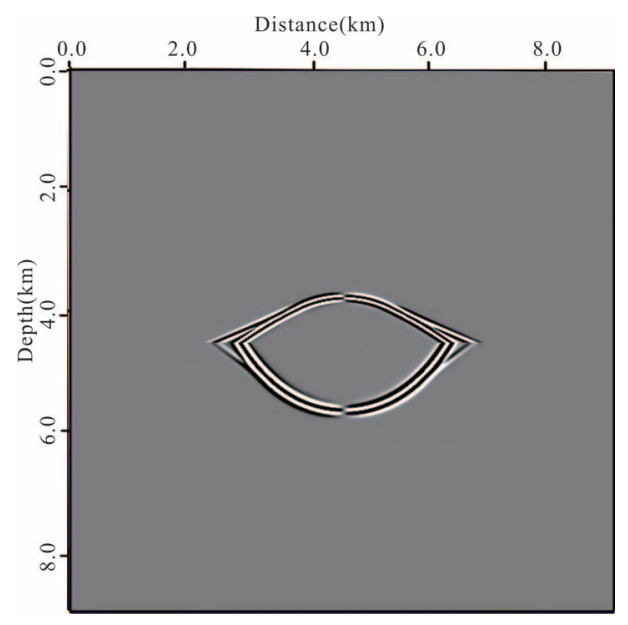

c)

d)

Figure 8. Elastic wavefield snapshots at $\mathrm{t}=0.8 \mathrm{~s}$ of a two-layer model by using the 4 th-order SGFD method and the 4 th-order ESGLFD method: (a) the $x$-component snapshot of the P-wave by the SGFD method; (b) the $x$-component snapshot of the P-wave by the ESGLFD method; (c) the $x$-component snapshot of the S-wave by the SGFD method; (d) the $x$-component snapshot of the S-wave by the ESGLFD method. Head waves are highlighted by the red arrows.

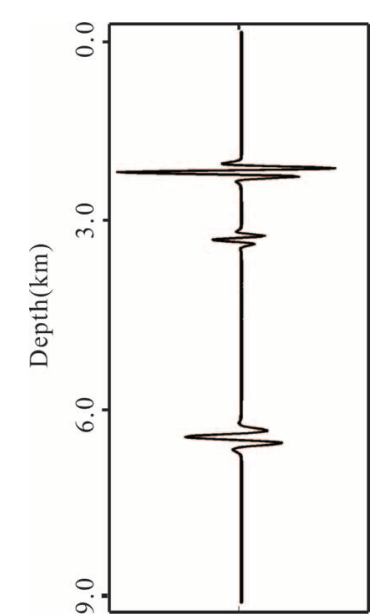

a)

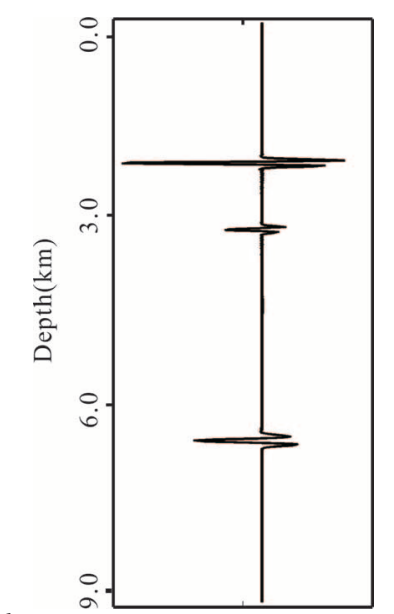

b)

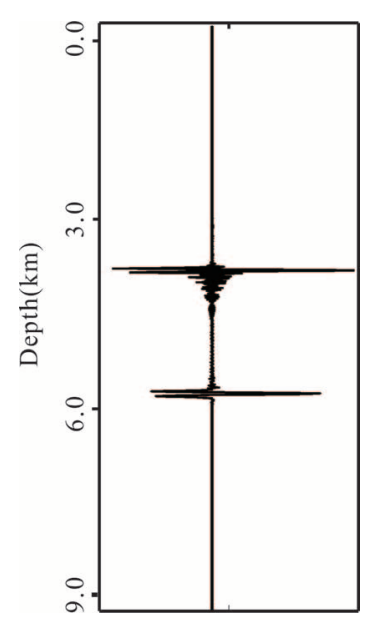

c)

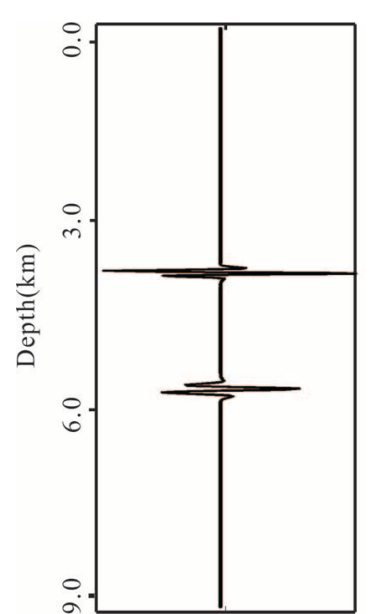

d)

Figure 9. Vertical slices extracted from the $x$-component snapshots (a) related to P-wave by the SGFD method, (b) related to P-wave by the ESGLFD method, (c) related to S-wave by the SGFD method, and (d) related to S-wave by the ESGLF D method at the distance of $3.0 \mathrm{~km}$. 


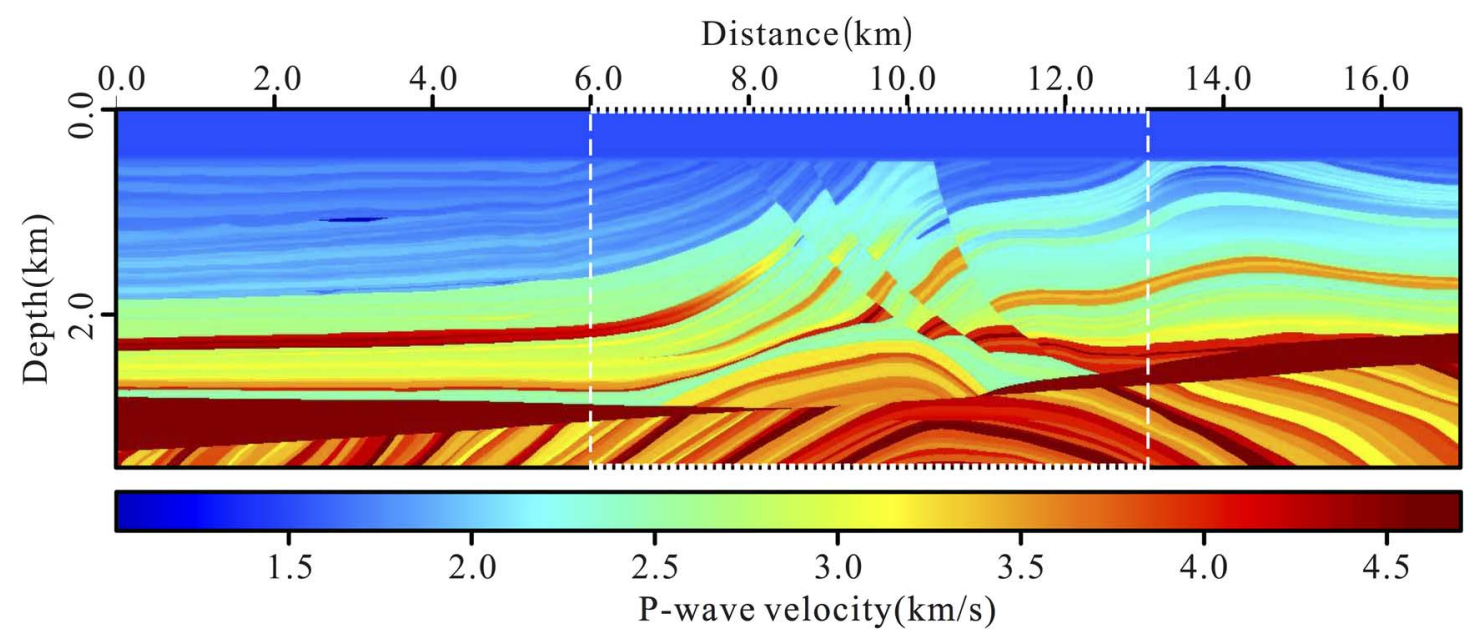

a)

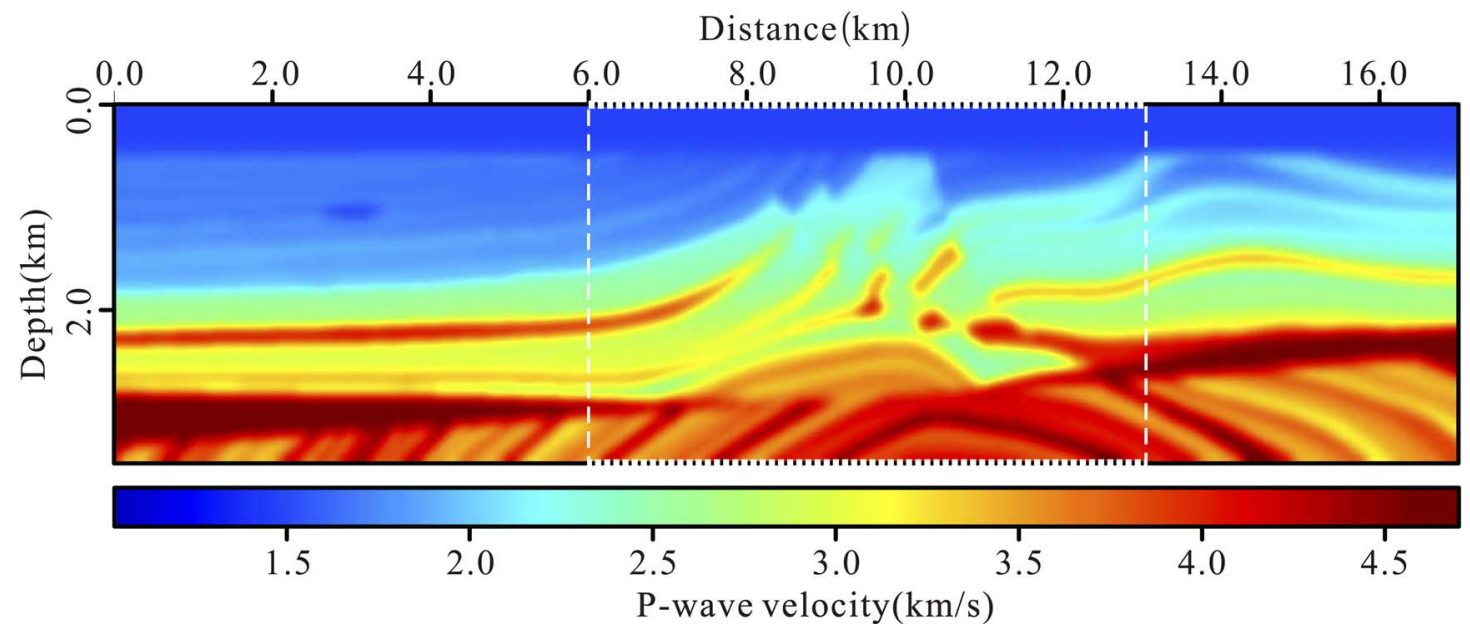

b)

Figure 10. The resampled (a) and smoothed (b) Marmousi2 model corresponding to P-wave velocity for elastic wave simulation and reverse time migration, respectively. The subset denoted by the dashed rectangle is used for elastic wave simulation.

exact coordinates of which are $(6.0,0) \mathrm{km},(13.0,0) \mathrm{km},(6.0,3.5) \mathrm{km}$, and $(13.0,3.5) \mathrm{km}$. Both the horizontal and the vertical space increments are $10 \mathrm{~m}$. The partial model is determined with a grid size of $700 \times 350$, and the Ricker wavelet is used as the explosive source term in this case, with the dominant frequency of $25 \mathrm{~Hz}$. The time sampling increment is $1 \mathrm{~ms}$. The PML boundary is implemented on the four sides of the model and has a thickness of $300 \mathrm{~m}$. The snapshots corresponding to P- and S-wave vector components at $0.8 \mathrm{~s}$ (as shown in Figure 11) are generated by the sixth-order ESGLFD method. The distinction between P- and S-waves can be clearly delineated with these snapshots. We could also see that the information of the $S$-wave is a very valuable part to depict the subtle structure of the complex velocity-variation model. Figure 12 shows two representative elastic common-source gathers corresponding to horizontal and vertical components, respectively. The direct waves have been removed and we can see that the reflection waves are without any visible numerical dispersion. The experiment verifies that the ESGLFD method can handle the complex media and provide the accurate wavefield for the subsequent RTM.

\subsection{Elastic Reverse Time Migration}

The 2D Marmousi2 model, as is shown in Figure 10, is used in this section for further RTM, in which the ESGLFD method serves as the forward and backward wavefield extrapolators. The geometry of the survey is from 


\section{Qizhen Du et al.}
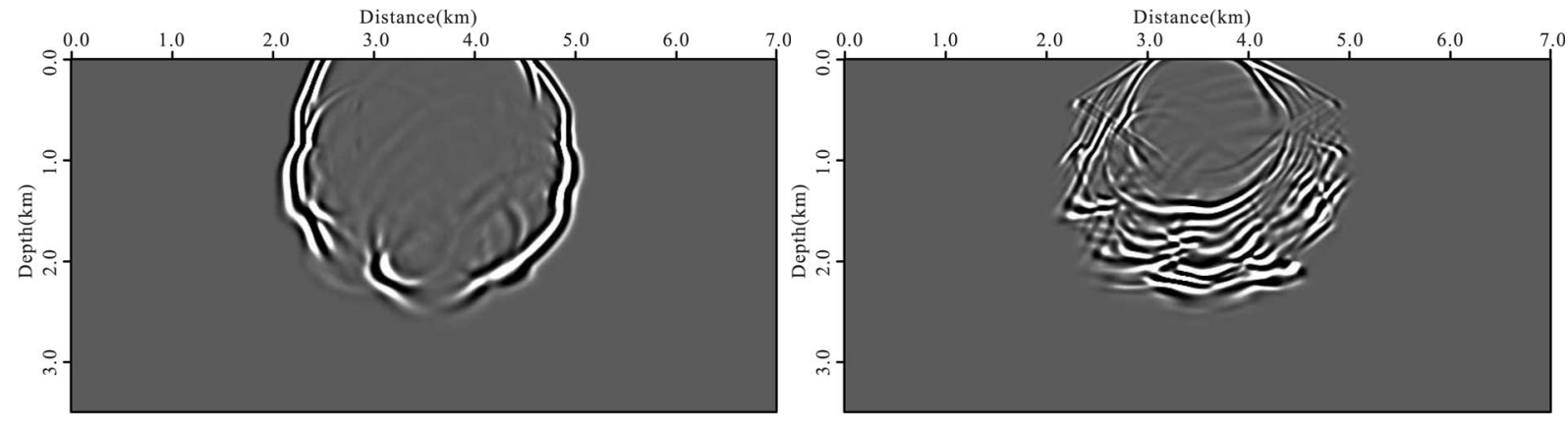

a)

b)
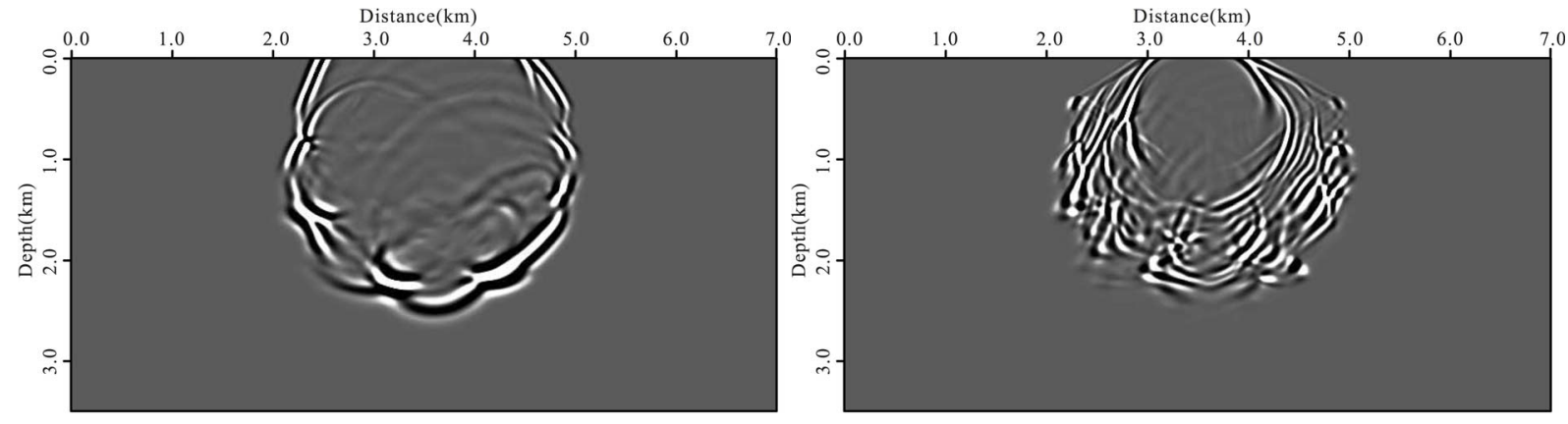

c)

d)

Figure 11. Snapshots corresponding to the horizontal component of the P-wave (a), the vertical component of the P-wave (b), the horizontal component of the S-wave (c) and the vertical component of the S-wave (d) at $0.8 \mathrm{~s}$ with the 6th-order ESGLFD method. The model used for elastic wave simulation is the subset, denoted by the dashed rectangle, of the resampled elastic Marmousi2, shown in Figure 9.

$0 \mathrm{~km}$ to $17.0 \mathrm{~km}$, in which a total number of the split-spread source is 340 with 700 receivers per shot. For each shot, one-half of the receivers are located on either side of the shot. All the sources and receivers are situated on the surface of the model, with the source spacing $50 \mathrm{~m}$ and receiver spacing $10 \mathrm{~m}$. Similarly, to the elastic wave simulation, the Ricker wavelet with the dominant frequency of $25 \mathrm{~Hz}$ is utilized. Total record time is $6 \mathrm{~s}$, with time sample increment of $1 \mathrm{~ms}$. Horizontal and vertical sampling intervals are both set at $10 \mathrm{~m}$. Additionally, PML with the thickness of $300 \mathrm{~m}$ on each side of the model is implemented. With regards to image condition, we utilize the scalar imaging conditions [Du et al., 2017], which obtain the scalar images using the normalized cross-correlation
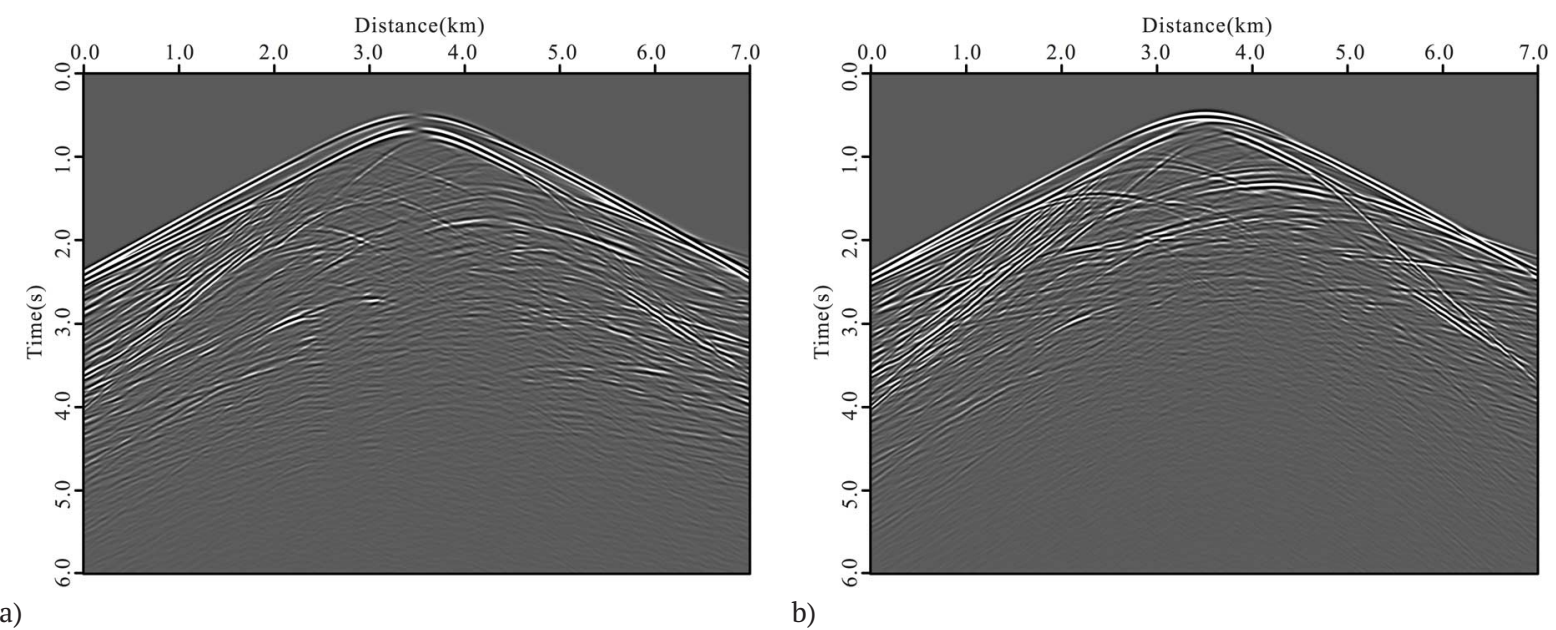

a)

b)

Figure 12. Elastic common-source gathers corresponding to the horizontal component (a) and the vertical component (b). 


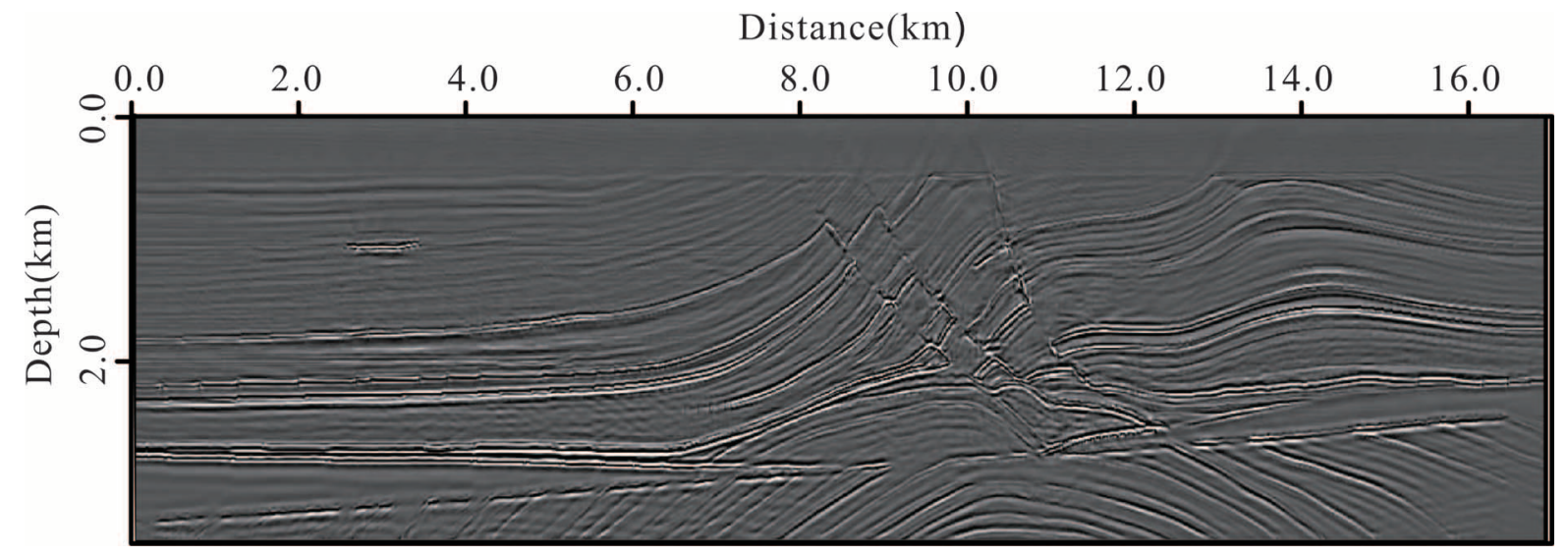

a)

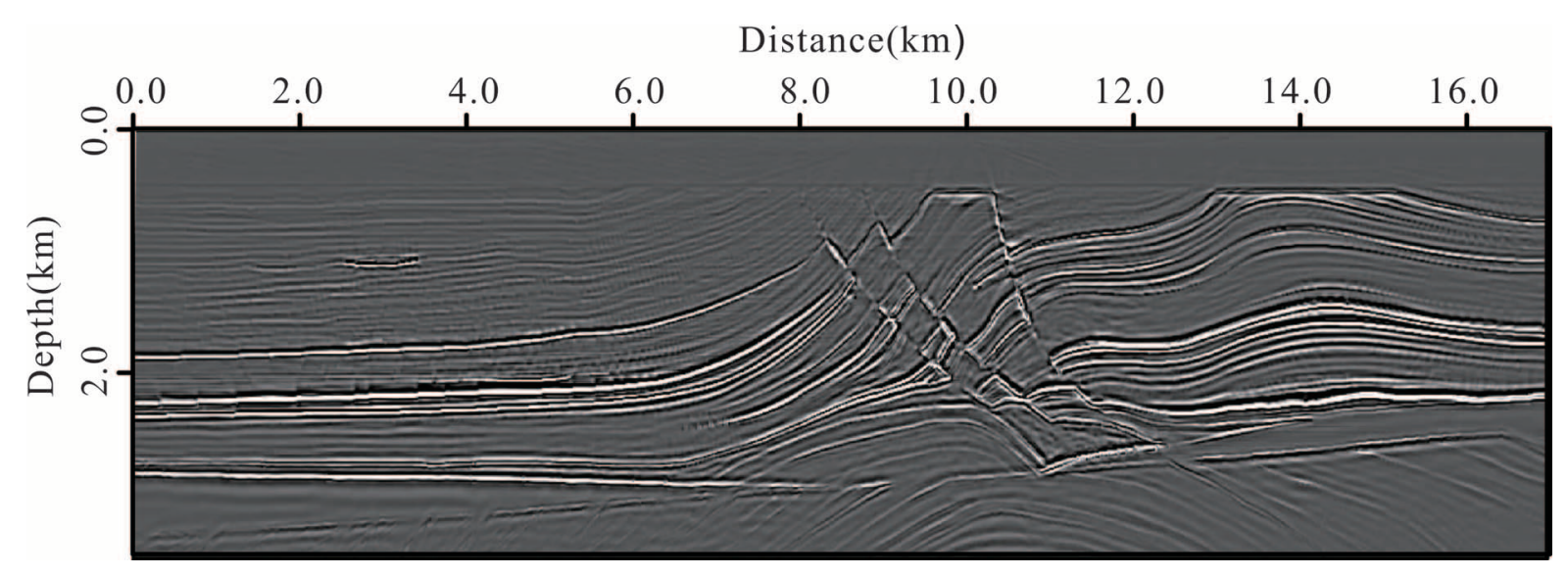

b)

Figure 13. The stacked PP image component (a), the stacked PS image component (b).

between the source-side wavefields and the receiver-side wavefields. This method eliminates the artifacts resulting from the crosstalk between different types of wave modes and avoids the polarity reversal for PS images, which may lead to destructive interferences in the processing of the adjacent source stack.

The final migration images, including the PP image component and PS component, are described in Figure 13. It can be observed that not only the stratoid and the flat structure but also the anticline and the faults appear clearly in all of these image profiles. Generally, these comparative image results shown in Figure 13 verify that the proposed method is sufficient to the function as an extrapolator to reconstruct the forward and the backward wavefields for elastic RTM.

\section{Conclusions}

In this work, a method of staggered-grid lowrank finite-difference is proposed to obtain a better representation of the elastic wave extrapolation. Numerical experiments on a two-layers model and the complex Marmousi2 model validate the precision and efficiency of the method. The following conclusions are achieved.

1. The basic assumption of the method is elasticity and linearity, which is composed of the dynamic and kinetic information of the primary and shear waves.

2. The method is derived on the basis of decomposition and plane wave theory, oriented to decompose the elastic wavefield to the pure-mode wavefield. The wavefield decomposition is the prerequisite of this method. 


\section{Qizhen Du et al.}

3. The elastic recursive time integration operator of the proposed method depends on the parameters of the elastic media and the simulation which can efficiently compensate the temporal accuracy loss.

4. The elastic lowrank finite-difference operators are optimal finite-difference operators and constructed in accordance with the spectral response in certain range of frequency. And the numerical tests indicate that the operators are capable of handling media with large velocity gradient.

5. The proposed method still follows the finite-difference scheme. In implementation, its efficiency will be a little lower than that of the regular staggered-grid finite-difference. That is because more memory space is needed to store the space-variant finite-difference coefficient and also more memory access will be introduced.

Acknowledgements. We appreciate the support of the National Science Foundation of China $(41930429,41774139)$ and the National Key Research \& Development Program of China (2017YFB0202903). And we appreciate the enlightening discussion of our related works with Chengfeng Guo, Sanyi Yuan, Qamar Yasin, Guanchao Wang and Xingyu Zhang. We thank the developers of Madagascar for providing the open-source software.

\section{References}

Aki K., Richards P.G. (2002). Quantitative seismology, University Science Books (2nd Edition).

Bérenger J.P. (1994). A perfectly matched layer for the absorption of electromagnetic waves, J. Comput. Phys. 114(2), $185-200$.

Bojarski N.N. (1985). The k-space formulation of the scattering problem in the time domain: An improved single propagator formulation, J. Acoust. Soc. Am. 71(2), 97 - 100.

Candes E., L. Demanet and L. Ying (2007). Fast computation of fourier integral operators, SIAM J. Sci. Comput. 29(6), 2464-2494.

Cheng J.B., T. Alkhalifah, Z.D. Wu, P. Zou, C. Wang (2016). Simulating propagation of decoupled elastic waves using low-rank approximate mixed-domain integral operators for anisotropic media, Geophysics 81(2), T63-T77.

Compani-Tabrizi B. (1986). K-space scattering formulation of the absorptive full fluid elastic scalar wave equation in the time domain, J. Acoust. Soc. Am. 79(4), 901-905.

Du Q.Z, Y.T. Zhu, J. Ba (2012). Polarity reversal correlation for elastic reverse time migration, Geophysics 77( 2), S31-S41.

Du Q.Z., X.F. Gong, M.Q. Zhang, Y.T., G. Fang (2014). 3D PS-wave imaging with elastic reverse-time migration, Geophysics 79 (5), S173-S184.

Du Q.Z., D. Han, S.A. Hou (2016). Lowrank Finite-difference Method for Elastic Wave Propagation, $78^{\text {th }}$ EAGE Conference \& Exhibition, Vienna, A.T., Extended Abstracts.

Du Q, Guo C F, Zhao Q, et al. (2017). Vector-based elastic reverse time migration based on scalar imaging condition, Geophysics 82(2), S111-S127.

$\mathrm{Du}$ X., P.J. Fowler, R.P. Fletcher (2013). Recursive integral time-extrapolation methods for waves: A comparative review, Geophysics 79(1), T9-T26.

Engquist B., L. Ying (2007). Fast directional multilevel algorithms for oscillatory kernels, SIAM J. Sci. Comput. 29(4), 1710-1737.

Engquist B., L. Ying (2009). A fast-directional algorithm for high frequency acoustic scattering in two dimensions, Comm. Math. Sci. 7(2), 327-345.

Etgen J., S. Brandsberg-Dhl (2009). The pseudo-analytical method: Application of Pseudo-Laplacians to acoustic acoustic and acoustic anisotropic wave propagation, $79^{\text {th }}$ SEG Meeting, Houston, USA, Expanded Abstracts, 2552-2556.

Fang G., S. Fomel, Q.Z. Du, J. Hu (2014). Lowrank seismic wave extrapolation on a staggered grid, Geophysics 79(3), T157-T168.

Finkelstein B., R. Kastner (2007). Finite-difference time domain dispersion reduction schemes, J. Comput. Phys. 221(1), 422-438.

Fornberg B., and G.B. Whitham (1978). A numerical and theoretical study of certain nonlinear wave phenomena, Philos. Trans. R. Soc. London 289, 373-404 
Fomel S., L. Ying, X. Song (2013). Seismic wave extrapolation using low rank symbol approximation, Geophys. Prospect. 61(3), 526-536.

Granger P.Y., M. Martin, J.L. Boelle, E. Ceragioli, F. Lefeuvre, E. Crouzy (2005). Autonomous 4C nodes used in infill areas to complement streamer data, deepwater case study, $75^{\text {th }}$ SEG Meeting, Houston, USA, Expanded Abstracts, 84-87.

Han D., Q.Z. Du, M.Q. Zhang, C.F. Guo, X.F. Gong (2016). Effects of staggered-grid and rotated staggered-grid on the wavefield separation in VTI media, Acta Seismol. Sin. 38(1), 111-121.

Han D., Q.Z. Du, G. Fang (2016). Elastic wave propagation on a staggered-grid using lowrank finite-difference method. 86th SEG Meeting, Dallas, USA, Expanded Abstracts, 3900-3904.

Li Z.C., H. Zhang, Q.M. Liu, W.G. Han (2007). Numerical simulation of elastic wavefield separation by staggered-grid high-order finite-difference algorithm (in Chinese), Oil Geophys. Prospect. 42(5), 510-515.

Liu Y., M.K. Sen (2009). A new time-space domain high-order finite-difference method for the acoustic wave equation, J. Comput. Phys. 228(23), 8779-8806.

Liu Y., M.K. Sen (2011). Scalar wave equation modeling with time-space domain dispersion-relation-based staggered-grid finite-difference schemes, Bull. Seismol. Soc. Am. 101(1), 141-159.

Ma D.T., G.M. Zhu (2003). P-and S-wave separated elastic wave equation numerical modeling (in Chinese), Oil Geophys. Prospect. 38, 482-486.

Martin G.S., R. Wiley, K.J. Marfurt (2006). Marmousi2: An elastic upgrade for Marmousi, The Leading Edge, 25: 156166.

Mast T.D., L.P. Souriau, D. Liu, M. Tabei, A.I. Nachman, and R.C. Waag (2001). A k-space method for large-scale models of wave propagation in tissue, IEEE Trans. Ultrason Ferroelectr. Freq. Control 48, 341-354.

Nguyen B.D., G.A. McMechan (2013). Excitation amplitude imaging condition for prestack reverse-time migration, Geophysics 78(1), S37-S46.

Pestana R.C., P. L. Stoffa (2010). Time evolution of the wave equation using rapid expansion method, Geophysics 75(4), T121-T131.

Pourjavid S., O.J. Tretiak (1992). Numerical solution of the direct scattering problem through the transformed acoustical wave equation, J. Acoust. Soc. Am. 91(2), 639-45.

Reshef M., D. Kosloff, M. Edwards, C. Hsiung (1988). Three-dimensional acoustic modeling by the Fourier method, Geophysics 53(9), 1175-1183.

Robertsson J.O., I. Moore, M. Vassallo, A.K. , D.J. Manen van, A. (2008). On the use of multicomponent streamer recordings for reconstruction of pressure wavefields in the crossline direction, Geophysics 73(5), A45-A49.

Song X., S. Fomel, L. Ying (2013). Lowrank finite-differences and low-rank Fourier finite-differences for seismic wave extrapolation, Geophys. J. Int. 193, 960-969.

Soubaras R., Y. Zhang (2008). Two-step explicit marching method for reverses time migration. $70^{\text {th }}$ EAGE Conference \& Exhibition incorporating SPE EUROPEC, Rome, I.T., 2214-4609.

Stewart R.R., J.E. Gaiser, R.J. Brown, D.C. Lawton (2003). Converted-wave seismic exploration: Applications, Geophysics 68(1), 40-57.

Stoffa P.L., R.C. Pestana (2009). Numerical solution of the acoustic wave equation by the rapid expansion method (REM)-A one step time evolution algorithm, $79^{\text {th }}$ SEG Meeting, Houston, USA, Expanded Abstracts, 2272-2276.

Sun J., S. Fomel, L. Ying (2015). Low-rank one-step wave extrapolation for reverse time migration, Geophysics 81(1), S39-S54.

Sun R., J.D. Chow, K.J. Chen (2001). Phase correction in separation P-and S-waves in elastic data, Geophysics 66(5), 1515-1518.

Tabei M., T.D. Mast, R.C. Waag (2002). A k-space method for coupled first-order acoustic propagation equations, J. Acoust. Soc. Am. 111(1), 53-63.

Tan S.R., L.J. Huang (2014). An efficient finite-difference method with high-order accuracy in both time and space domains for modelling scalar-wave propagation, Geophys. J. Int. 197, 1250-1267.

Virieux J. (1984). SH-wave propagation in heterogeneous media: velocity-stress finite-difference method, Geophysics 49(11), 1933-1942.

Virieux J. (1986). P-SV wave propagation in heterogeneous media: Velocity-stress finite-difference method, Geophysics 51(4), 889-901.

Voynov O.Y., V.I. Golubev, M.S. Zhdanov, I.B. Petrov (2018). Migration of elastic wavefield using adjoint operator and 


\section{Qizhen Du et al.}

Born approximation. Innovations in Wave Modelling and Decision Making, SIST Series, Volume 90, Springer Switzerland, Chapter 8, P. 219-240.

Wang W., and G.A. McMechan (2015). Vector-based elastic reverse-time migration, Geophysics 80, S245-S258.

Wards B., G. Margrave, M. Lamoureux (2008). Phase-shift time-stepping for reverse-time migration. 78thSEG Meeting, Las Vegas, USA, Expanded Abstracts, 2262-2266.

Yan J., P. Sava (2008). Isotropic angle-domain elastic reverse-time migration, Geophysics 73(6), S229-S239.

Yan J., P. Sava (2009). Elastic wave-mode separation for VTI media, Geophysics 74(5), WB19-WB32.

Yan R., X.B. Xie (2012). An angle-domain imaging condition for elastic reverse time migration and its application to angle gather extraction, Geophysics 77(5), S105-S115.

Yuan S., S. Wang, W. Sun, L. Miao, Z. Li (2014). Perfectly matched layer on curvilinear grid for the second-order seismic acoustic wave equation, Explor. Geophys. 45, 94-104.

Zhang Q., G.A. McMechan (2010). 2D and 3D elastic wavefield vector decomposition in the wavenumber domain for VTI median, Geophysics 75(3), D13-D26.

Zhang Y., G. Zhang (2009). One-step extrapolation method for reverse time migration. Geophysics 74(4), A29-A33. 